\title{
1 Rare and undersampled dimorphic basidiomycetes
}

3 Kachalkin, A.V. †; Turchetti, B. †; Inácio, J. †; Carvalho, C.; Mašínová, T.; Pontes, A.; Röhl, 4 O.; Glushakova A.M.; Akulov, A.; Baldrian, P.; Begerow, D.; Buzzini, P.; Sampaio, J.P.;

5 Yurkov A.M.*

6

$7 \quad$ * corresponding author: Leibniz Institute DSMZ - German Collection of Microorganisms and

8 Cell Cultures, Inhoffenstrasse 7B, 38124 Braunschweig, Germany; andrey.yurkov@dsmz.de

$9 \dagger$ equal contribution

Affiliations:

Kachalkin, A.V.;

Lomonosov Moscow State University, 119991 Moscow, Russia

All-Russian Collection of Microorganisms (VKM), G. K. Skryabin Institute of Biochemistry and Physiology of Microorganisms, Russian Academy of Sciences, 142290 Pushchino, Russia

Glushakova A.M.;

Lomonosov Moscow State University, 119991 Moscow, Russia

Turchetti, B.; Buzzini, P.;

Department of Agricultural, Food and Environmental Sciences \& Industrial Yeasts Collection

Inácio, J.;

School of Pharmacy and Biomolecular Sciences, University of Brighton, Lewes road,

Brighton BN2 4GJ, United Kingdom

Global Health and Tropical Medicine, GHTM, Unit of Medical Microbiology, Instituto de Higiene e Medicina Tropical, IHMT, Universidade Nova de Lisboa, UNL, Rua da Junqueira 100, 1349-008 Lisbon, Portugal

Carvalho, C.; Pontes, A.; Sampaio, J.P.;

UCIBIO-REQUIMTE, Departamento de Ciências da Vida, Faculdade de Ciências e

Mašínová, T.; Baldrian, P.;

31 Laboratory of Environmental Microbiology, Institute of Microbiology of the CAS, 14220

32 Praha 4, Czech Republic

33 Akulov, A;

V. N. Karazin Kharkiv National University, 61022 Kharkiv, Ukraine 
Röhl, O.; Begerow, D.;

36 Geobotany, Faculty of Biology and Biotechnology, Ruhr-University Bochum, 44801

37 Bochum, Germany

38 Yurkov A.M.;

39 Leibniz Institute DSMZ - German Collection of Microorganisms and Cell Cultures, 38124

40 Braunschweig, Germany

\section{Abstract}

The diversity of yeasts has grown rapidly as the discovery of new species has benefited from intensified sampling and largely improved identification techniques. An environmental study typically reports the isolation of yeast species, some of which are new to science. Rare species represented by a few isolates often do not result in a taxonomic description. Nucleic acid sequences from these undescribed yeasts remain in public sequence databases, often without a proper taxonomic placement. This study presents a constrained phylogenetic analysis for many rare yeasts from unpublished but publicly available DNA sequences and from studies previously conducted by the authors of this work. We demonstrate that single isolates are an important source of taxonomic findings such as including new genera and species. Independent surveys performed during the last 20 years on a large geographic scale yielded a number of single strains, which were proved to be conspecific in the phylogenetic analyses presented here. The following new species were resolved and described: Vustinia terrea Kachalkin, Turchetti \& Yurkov gen. nov. et sp. nov., Udeniomyces caspiensis Kachalkin sp. nov., Udeniomyces orazovii Kachalkin sp. nov., Tausonia rosea Kachalkin sp. nov., Itersonilia diksonensis Kachalkin sp. nov., Krasilnikovozyma fibulata Glushakova \& Kachalkin, Kwoniella fici Turchetti sp. nov., Heterocephalacria fruticeti f.a. Carvalho, Roehl, Yurkov \& Sampaio sp. nov., Heterocephalacria gelida f.a. Turchetti \& Kachalkin sp. nov., Heterocephalacria hypogea f.a. Carvalho, Roehl, Yurkov \& Sampaio sp. nov., Heterocephalacria lusitanica f.a. Inacio, Carvalho, Roehl, Yurkov \& Sampaio sp. nov., Piskurozyma arborea Yurkov, Kachalkin, Mašínová \& Baldrian sp. nov., Piskurozyma silvicultrix Turchetti, Mašínová, Baldrian \& Yurkov sp. nov., Piskurozyma stramentorum Yurkov, Mašínová \& Baldrian sp. nov., Naganishia nivalis Turchetti sp. nov., Yurkovia nerthusi Yurkov \& Begerow, sp. nov. In addition, two new combinations were proposed Krasilnikovozyma curviuscula (Babeva, Lisichkina, Reshetova \& Danilevich) Yurkov, Kachalkin \& Sampaio comb. nov., Hannaella taiwanensis (F.L. Lee \& C.H. Huang) Yurkov comb. nov. The order Cyphobasidiales T. Spribille \& H. Mayrhofer is rejected in favour of the older name Erythrobasidiales R. Bauer, Begerow, J.P. Sampaio, M. Weiss \& Oberwinkler. Other potential novel species identified in this paper await future description. Phylogenetic placement of yet unpublished sequences is believed to facilitate species descriptions and improve classification of yeasts from environmental sequence libraries.

Keywords: 1 new genus, 17 new species, 2 combinations, yeasts, taxonomy, 


\section{Introduction}

Our knowledge of a yeast stage of members of the phylum Basidiomycota dates back to the 19th century and the pioneering work of Brefeld, who observed the germination of teliospores of the corn smut Mycosarcoma maydis with budding yeast-like cells (reviewed by Oberwinkler 2017). Subsequent mating experiments confirmed the presence of the filamentous-yeast switch in several smuts (reviewed by Oberwinkler 2017). Later, Kluyver and van Niel (1927) pointed to the similarity between forcibly ejected buds of a red yeast belonging to the genus Sporobolomyces and basidiospores of basidiomycetes. The most conclusive evidence of a basidiomycete connection was the discovery of mating and a sexual state in strains of Rhodotorula glutinis by Banno $(1963,1967)$, followed by the discoveries of teleomorphs of several yeasts and description of the genera Filobasidiella, Cuniculitrema and Bulleromyces. A number of morphological, biochemical, ultrastructural, and physiological criteria indicated the basidiomycetous affinity of many asexual yeasts. These characters include a positive diazonium blue B reaction, urease activity, enteroblastic mode of budding, presence of ballistoconidia, red carotenoid pigments, a lamellate cell wall ultrastructure, presence of a dolipore septum (in hyphae), the biochemical composition of the cell wall, and a high GC content of genomic DNA (Boekhout et al. 2011; Kurtzman and Boekhout 2017). However, evolutionary relationships between yeasts, whether asexual or sexual, and teleomorphic filamentous taxa remained unknown. Early molecular evolutionary studies of 5S rRNA distinguished two phylogenetic lineages, Agaricomycotina and Pucciniomycotina, and these results correlated well with septal ultrastructure (reviewed by Kurtzman and Boekhout 2017; Oberwinkler 2017). Later, studies of the SSU rRNA gene revealed the third lineage of basidiomycetes, the Ustilaginomycotina.

Studies of dimorphic heterobasidiomycetes followed two different directions (reviewed by Begerow et al. 2017). One approach taken by traditional mycologists, who sampled fungi in the field and investigated them in the laboratory. From these studies we know that sexual structures of some mycoparasites (e.g. Tremella, Rhynchogastrema, Trimorphomyces) and plant parasites (e.g. Microbotryum, Mycosarcoma) germinate with yeast states. Another approach was undertaken by yeast researchers and included mating experiments to obtain teleomorphic states on laboratory media (e.g. Curvibasidium, Leucosporidium, Papiliotrema, and Rhodosporidium) and the subsequent description of the relevant morphological characters, including basidial and hyphal morphology. It transpired that species commonly considered as yeasts form a sexual cycle ex situ and display features previously described as an adaptation to a parasitic lifestyle, for example appressoria, colacosomes and haustoria.

Yeasts were among the first fungi for which nucleic acids were sequenced and phylogenetically analysed, and their presence in all three subphyla in Basidiomycota was demonstrated by many molecular phylogenetic studies (reviewed by Kurtzman and Boekhout 2017). Pioneering studies from the 1980s showed that sexual and asexual taxa are intermixed in many clades suggesting that morphological dimorphism is a common feature among basidiomycetous yeasts. Yeasts were also the first group of fungi subjected to DNAbarcoding using ribosomal gene fragments such as LSU rRNA gene (Fell et al. 1995, 2000; Begerow et al. 1997; Fonseca et al. 2000; Sampaio 2004) and ITS (Scorzetti et al. 2002). 
These studies provided a solid background for fast and reliable identification of yeasts and facilitated the discovery of new species. Although the number of known yeast species in the Agaricomycotina and Pucciniomycotina was growing rapidly, the number of available sequences of teleomorphic taxa remained low (Fell et al. 2000; Millanes et al. 2011; Liu et al. 2015a; Wang et al. 2015b). Recent changes in fungal taxonomic rules have resulted in a unified classification system for naming sexual and asexual taxa, including lineages containing yeasts. Consequently, large polymorphic and polyphyletic anamorphic genera like Bullera, Cryptococcus, Rhodotorula, Sporobolomyces and Trichosporon were reclassified, which resulted in 35 new genera and 296 new combinations (Liu et al. 2015a; Wang et al. 2015b). Similarly, several teleomorphic species of Cystobasidium, Tremella and Syzygospora distantly related to clades that contain type species were reclassified (Liu et al. 2015a; Wang et al. 2015b; Millanes et al. 2016; Spirin et al. 2018). These studies demonstrated that undersampling and the lack of reference sequences hamper both taxonomical works and biodiversity assessments. It has been also shown that the lack of reference sequences and inaccessibility of material (culture or specimen) can result in taxonomic redundancy, as exemplified by the yeast genus Bandoniozyma (Tremellomycetes), which coincided with Rhynchogastrema, an earlier discovered, but not sequenced, filamentous genus (Liu et al. 2015a).

135 To date, most of our knowledge of diversity in Cystobasidiomycetes, Microbotryomycetes and Tremellomycetes is derived from studies reporting the discovery of new yeast species (Liu et al. 2015; Wang et al. 2015; Kurtzman and Boekhout 2017). Yeasts are isolated worldwide from a multitude of habitats and substrates (Peter et al. 2017). Many of them remain, however, undescribed because of an unclear phylogenetic placement or because they are known from a limited number of isolates (e.g., Seifert and Rossman 2010). Constrained phylogenetic analyses of LSU rRNA proved their usefulness and identified potential new species clustering in already recognized (sometimes monotypic) lineages (Liu et al. 2015a; Wang et al. 2015b). A few from these potential new species have been described recently (e.g., Yurkov et al. 2016a), and others still await a description in spite of having been discovered for a long time. Moreover, every new biodiversity study adds a few potential novel yeasts to the already existing pool of unnamed and unplaced species. Sometimes the number of potential novel yeasts reported in a study can be as large as $25-30 \%$ of the total diversity (e.g., Yurkov et al. 2012a, 2016b; Mašínová et al. 2017a, 2017b). Thus, it becomes challenging for researchers to maintain an overview of the ever-growing diversity of undescribed yeasts.

151 The aim of this paper is to provide updated phylogenies of yeasts in Agaricomycotina and Pucciniomycotina, and identify new clades in these groups. The work includes previously isolated but yet undescribed species and more recent isolates obtained from a number of studies performed by us. Many of these species belong to the so-called heterobasidiomycetes, a group of fungi extensively studied by Franz Oberwinkler and his collaborators, including Robert Bauer and Robert Bandoni. Also, several strains from the former collection of the University of Tübingen served a reference for teleomorphic taxa for which no sequence was available. Several new species and one new genus identified in this study are described. 
160 Sampling and isolation of yeasts have been independently performed in previous studies.

161 Isolations from soils in Czech Republic, Germany and Portugal followed protocols described

162

163

164

165

166 by Mašínová et al. (2017b) and Yurkov et al. (2012a, 2016b), respectively. Strains from Russia were obtained using sampling and isolation protocols described by Glushakova et al. (2015). Yeasts on plant material in Portugal were studied by Inácio et al. (2002) and Inácio (2003). Isolations from glacial sampling and plant materials in Italy followed protocols described in Turchetti et al. (2013) and Franca et al. (2016).

Sequences of D1/D2 domains of the 26S rRNA gene (LSU) were aligned into multiple sequence alignments, previously used by Liu et al. (2015a) and Wang et al. (2015b), by utilising MAFFT online service (version 7) and --add and --keeplength functions (Katoh et al. 2017). New sequences were either obtained from public databases or produced in previous studies. Resulting alignments contained a total of 860 sequences, 301 for Pucciniomycotina and $\underline{59}$ for Agaricomycotina. Trees were constructed with raxmlGUI 1.5b software using the maximum-likelihood algorithm and GTRGAMMA (GTR substitution model with gammadistributed rate heterogeneity) model with 100 bootstrap replicates; topological constrains were enforced as was described previously by Liu et al. (2015a) and Wang et al. (2015b). A parsimony network was constructed from aligned LSU and ITS sequences with the program TCS 1.21 (Clement et al. 2000) using a 95\% connection limit and gaps treated as missing data. A fragment of the gene encoding translation elongation factor 1 alpha (TEF1) was amplified and sequenced following the previously described protocols (Yurkov et al. 2015a, 2015b; Spirin et al. 2018). Multiple sequence alignments were performed with the genomic sequences using online version of MAFFT algorithm (Katoh et al. 2017). Phylogenetic relationships were inferred from a concatenated dataset of the ITS region, LSU rRNA and TEF1 genes as described above.

Assimilation tests were performed on solid and in liquid media following the procedures described by Kurtzman et al. (2011), with API 50CH test strips (bioMérieux) and YT and FF MicroPlates (Biolog) as described previously (Yurkov et al. 2017; Mašínová et al. 2018). For observing micro-morphology, cultures were grown at $10-22^{\circ} \mathrm{C}$ on YM, PD, GPY and $0.5 \%$ glucose-YNB agars and studied with phase-contrast optics.

\section{Results and discussion}

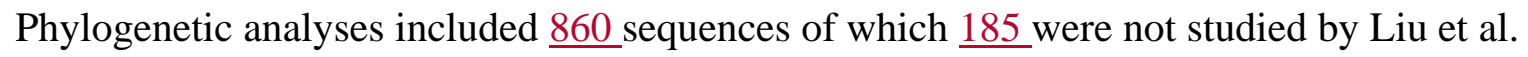
(2015a) or Wang et al. (2015b). The overall topology was consistent with the two previous constrained LSU analyses (Liu et al. 2015a; Wang et al. 2015b), although several lineages were enlarged with potential novel species (Figs. 1, 2). We discuss some of these clades below. 


\section{Cystofilobasidiales}

201

The order Cystofilobasidiales comprises two families, Mrakiaceae and Cystofilobasidiaceae (Liu et al. 2015a). The study of Liu et al. (2015a) indicated a number of sequences corresponding to potential new species in the genera Itersonilia, Krasilnikovozyma, Tausonia and Udeniomyces. Also, two novel Cystofilobasidium species were recently described from Mediterranean soils (Pontes et al. 2017). Three yeasts isolated from cold soils in Russia (Altay region), Italy (Alps) and Kyrgyzstan (Byshkek) showed highly similar sequences and were placed close to the genus Krasilnikovozyma (Fig. 3). Our phylogenetic analyses based on LSU, ITS and TEF1 suggest these yeasts are likely to represent a new genus in Mrakiaceae (see Taxonomy below).

The genus Krasilnikovozyma was proposed by Liu et al. (2015a) to accommodate members of the so-called Cryptococcus huempii clade, which also included the species Mrakia curviuscula and Cryptococcus tahquamenonensis. C. huempii and M. curviuscula were sequenced from the material available at the CBS culture collection (strains CBS 8168 and CBS 9136, respectively) and showed identical D1/D2 sequences (GenBank AF189844 and EF118826, respectively) suggesting these yeasts belong to the same species. ITS sequence of Mrakia curviuscula strain CBS 9136 was first published by Liu et al. (2015b). Because both LSU (see above) and ITS (GenBank KY103891 and KY103892, respectively) sequences of the two species were identical, Mrakia curviuscula was considered to be a taxonomic synonym of Krasilnikovozyma huempii by Liu et al. (2015a, 2015b).

\section{Cryptococcus tahquamenonensis (presently Krasilnikovozyma tahquamenonensis) was} described based on a single strain from soil; its LSU sequence (KM408125) shows 98\% similarity to the type strain of Cryptococcus huempii (AF189844) and differs in 9 substitutions (including two N base calls in and 1 gap in KM408125 using GenBank Blastn). The similarity of the ITS sequences of the two species is 92\% (GenBank KM384610 and AF444322), thus allowing a proper differentiation of these yeasts with either LSU or ITS sequencing.

The type material of Mrakia curviuscula strain Oz-358 was preserved in the yeast collection of the Lomonosov Moscow State University as KBP Y-3618 (holotype) and deposited (extype) in the VKM collection as VKM Y-2953 (Babjeva et al. 2002). The original culture Oz358, from which the holotype Oz-358 was derived, was revived in 2003 and sub-cultured, following a request from the Portuguese Yeast Culture Collection. The progeny of Oz-358 was preserved in and later transferred to PYCC as PYCC 5836. Sequencing, performed independently, of cultures derived from the type material, i.e. VKM Y-2953 (GenBank MK244628) and PYCC 5836 (GenBank MF372143, MF372124), demonstrated that the two strains share identical LSU and ITS sequences, which showed high similarity to sequences of the type strain of Krasilnikovozyma (Cryptococcus) tahquamenonensis (ITS: KM384610, LSU: KM408125). Specifically, comparison of ITS sequences showed 3 mismatches in ITS2, corresponding to 1 base pair difference and two gaps. Our results suggest that the presumptive ex-type CBS 9136 is not identical to two other ex-types VKM Y-2953 and PYCC 5836. In 
our opinion, VKM Y-2953 and PYCC 5836 are the correct ex-type cultures, whereas CBS 9136 is a strain of K. huempii. Sequencing of ex-types VKM Y-2953 and PYCC 5836 suggests that $M$. curviuscula is conspecific with $K$. tahquamenonensis. However, Mrakia curviuscula Babeva, Lisichkina, Reshetova \& Danilevich (2002) is older than Cryptococcus tahquamenonensis Q.M. Wang, A.B. Hulfachor, K. Sylvester \& C.T. Hittinger (2015) and, thus, has a taxonomic priority. Therefore, we resurrect and recombine Mrakia curviuscula Babeva, Lisichkina, Reshetova \& Danilevich (2002) as Krasilnikovozyma curviuscula comb. nov. and put Krasilnikovozyma tahquamenonensis (Q.M. Wang, A.B. Hulfachor, K. Sylvester \& C.T. Hittinger) A.M. Yurkov (2015) in taxonomic synonymy with the former (see Taxonomy).

A GenBank search resulted in a number of sequences labelled as Mrakia frigida and Mrakia gelida. Many of these strains have ITS sequences that differ from those of type strains of $M$. frigida and M. gelida. Although nucleotide sequences of the ITS region were successfully used to delimit species in the genus Mrakia, these results suggest that additional genes should be used in the future to reassess the most useful criteria for species delimitations in this genus.

\section{Tremellales}

Family Bulleraceae. A single-species lineage represented by Cryptococcus mujuensis in a 7gene phylogeny (Liu et al. 2015b) was enlarged with two more species in a LSU-phylogeny (Liu et al. 2015a). These tree species were transferred to the newly erected genus Fonsecazyma (Liu et al. 2015a). This study also indicated that this clade contains four potential new species. Yeasts of this genus have been isolated from diverse regions mostly from plant material (Herzberg et al. 2002; Inácio 2003; Mittelbach et al. 2016; Sylvester et al. 2015). The Tremella clade I (sensu Millanes et al. 2011) comprises not yet re-classified species of the genera Tremella and Sirobasidium, some of which are also known from living cultures. We identified two more cultures in public collections, namely a yet undescribed Tremella species represented by two cultures, DSM 104578 (Germany, FO 24396) and NBRC 32520 (Japan). Another species in this clade, closely related to Cryptococcus cuniculi pro. temp., is represented by strain KBP Y-5716 (MH697756). The genus Pseudotremella was proposed to accommodate the well-supported clade including T. moriformis by Liu et al. (2015a). These authors also reported some inconsistency between sequences of $T$. indecorata, which clustered in Pseudotremella and Tremella clade I. In addition to the aforementioned inconsistency, we found some heterogeneity between sequences of specimens identified as $P$. moriformis (Fig. 1스). Specifically, Tremella moriformis specimen UBC F13838 (= RB284b, sometimes wrongly cited as RB2846 and RJB2846), collected by Wells from UC Davis (USA), was sequenced by Chen in Tübingen, Germany (GenBank AF042426, AF042244). Sequences of this specimen were highly similar to another recently collected Tremella specimen CWU (MYC) 136 from Kharkiv (Ukraine) and a yeast isolate from Italy (DBVPG 10729, GenBank MK634540) (Fig. 1스). Phylogenetic analyses demonstrated that they are

279 placed in the genus Pseudotremella but very distant from the reference specimen of $P$. 280 moriformis_UBC F13868 (= CBS 7810, GenBank AF075493, AF444331) used by Liu et al. 
281 (2015a). Another sequence representing a potentially conspecific isolate was found in

282

283 GenBank (MG190052); this yeast was isolated from an insect frass on an olive tree in South Africa.

Family Carcinomycetaceae. The mycoparasitic genus Carcinomyces (Oberwinkler \& Bandoni 1982) presently comprises two sexual and one asexual species. Our analysis identified another, yet undescribed, species in this genus isolated from an insect gallery in the USA (Fig. $1 \underline{A})$.

Family Cryptococcaceae. Our analyses revealed several potential new species in the genera Kwoniella and Cryptococcus from soil (Yurkov et al. 2016b, Mašínová et al. 2018), plant (Glushakova and Kachalkin 2017) and insect sources (Fig. 1).

Family Phaeotremellaceae. Gelidatrema, a monotypic genus proposed by Liu et al. (2015a), was expanded with an additional in our phylogenetic analysis (Fig. 1스). The recently described from a microbial mat in the Canadian High Arctic Gelidatrema psychrophila was previously observed by Turchetti et al. (2013). This culture, though the sequence was available for in Genbank (KC433781), was not considered in the description by Tsuji et al. (2018).

Family Sirobasidiaceae. The genus Fibulobasidium is distinguished from closely related Sirobasidium magnum, based on its unusual basidium development (Bandoni 1979). The genus received strong support in recent phylogenetic analyses (Liu et al. 2015a). Sirobasidium magnum (Liu et al. 2015a) and Sirobasidium japonicum (Liu et al. 2015b) were placed sister to the genus Fibulobasidium (Fig. 1B). In the present study, we identified a new closely related sub-clade represented by seven yeasts (Fig. 1ㅡ). These strains originate from diverse habitats and regions, all of which are characterized by a substantial water limitation, i.e. from Mediterranean soils, Greenland sandstone, moss-dominated desert soil crusts, grape berries and floral nectar (Cadez et al. 2010; Selbman et al. 2014; Mittelbach et al. 2015; Yurkov et al. 2016b). A sequence of an unspecified Sirobasidium sp. (GenBank LC203429) formed a wellsupported cluster with the genus Fibulobasidium (Fig. 1B), while S. intermedium is placed in Tremella clade I (Fig. 1A). Delimitation of the two genera will be difficult in the future because the sequence of the type species of the genus Sirobasidium, S. rubrofuscum (syn. S. sanguineum, Dämon and Hausknecht 2002), is not yet available.

Family Tremellaceae. The genus Tremella was re-defined to include only members of the $T$. mesenterica clade. A new lineage consisting of three isolates from soils and insect-related sources was identified close to the Tremella clade, though statistical support for this placement was low (Fig. 1ㅡㅡ).

Family Trimorphomycetaceae. The monotypic genus Sugitazyma was proposed to accommodate Bullera miyagiana (Liu et al. 2015a). Our results show that several yeasts were placed close to Sugitazyma miyagiana and Tremella parmeliarum, though statistical support for this clustering was low (Fig. 1ㅡ). These yeasts were isolated from soils (Czech Republic, Portugal), an insect (Bulgaria) and a plant (Taiwan). Two strains independently isolated in Serra da Arrábida, Portugal (Inácio 2003; Yurkov et al. 2016b) represent potentially new 
species, which were placed in the genus Saitozyma, though statistical support for this placement was low (Fig. 1B).

\section{Filobasidiales}

After recent re-classification of Cryptococcus species in Filobasidiales, yeasts in this lineage are accommodated in two existing (i.e. Filobasidium, Syzygospora), three new (i.e. Goffeauzyma, Piskurozyma, Solicoccozyma) and two resurrected (i.e. Heterocephalacria, Naganishia) genera (Liu et al. 2015a).

Family Filobasidiaceae. The genus Filobasidium has been restricted to the clades Magnus and Floriforme (sensu Fonseca et al. 2000, 2011; Scorzetti et al. 2002), which contain the type species F. floriforme (Liu et al. 2015a). Similarly, Liu et al. (2015a) resurrected the genus Naganishia, with the type species $N$. globosa, to accommodate members of the Albidus clade of the order Filobasidiales (sensu Fonseca et al. 2000, 2011; Scorzetti et al. 2002). Sixteen species are presently accepted in the genus considering the latest member, Naganishia qatarensis, isolated from a hypersaline marine environment (Fotedar et al. 2018). Our analysis revealed a new member of the genus isolated from cold environments. The study by Liu et al. (2015a) revealed four potential new species in the genus Heterocephalacria. Our analyses expand the number of yet undescribed species in this genus to eight (Figs. 1, 4). According to the available literature and GenBank records, these species were isolated from Mediterranean soils and plants (Inácio 2003; Yurkov et al. 2016b), supraglacial sediments (Turchetti et al. 2013), boreal swamp plants, tundra plants, and New Zealand soil.

Family Piskurozymaceae. The genus Piskurozyma was proposed to accommodate the wellsupported Cylindricus clade of the order Filobasidiales (Scorzetti et al. 2002; Fonseca et al. 2011), a single-species lineage F. capsuligenum ${ }_{2}$ and the mycoparasite Syzygospora sorana. The analysis performed by Liu et al. (2015a) showed two well-supported sub-clades in the genus, one comprising members of the Cylindricus clade and another one represented by $P$. fildesensis and two undescribed species, later described as P. yama and P. tuonelana (Yurkov et al. 2016b). Our study substantially expanded the size of the genus with seven potentially new species as suggested by phylogenetic analyses (Figs. 1, 5).

\section{Pucciniomycotina}

352 Similarly to studies in Tremellomycetes, recent taxonomic changes in yeasts belonging to

353 Cystobasidiomycetes and Microbotryomycetes were directed towards the re-classification of 354 large polyphyletic genera such as Bensingtonia, Rhodotorula, and Sporobolomyces (Yurkov 355 et al. 2015; Wang et al. 2015b).

356 In the Cystobasidiales, sequencing and subsequent phylogenetic analyses showed that the 357 parasitic lichen-inhabiting teleomorphic genus Cystobasidium is polyphyletic (Yurkov et al. 358 2015b; Millanes et al. 2016) and distributed between two clades. The first clade, containing 359 the type species $C$. fimentarium, also includes numerous yeast species from the so-called 360 Rhodotorula minuta clade (Yurkov et al. 2015b). The second clade ${ }_{2}$ with C. hypogymniicola 
B61

and C. usneaicola $a_{2}$ is placed outside Cystobasidiales and to Cyrenella elegans in the Erythrobasidiales (Fig. 2). It is important to document that Cyrenella elegans was not included in the phylogenetic analyses that addressed the taxonomic position of the genus Cyphobasidium (Millanes et al. 2016; Spribille et al. 2016). Below we discuss taxonomy of this genus in more detail. Recent studies expanded Cystobasidium and the closely genus Occultifur with three and four new yeast species, respectively (Wang et al. 2015b; Šibanc et al. 2018; Turchetti et al. 2018).

In the Microbotryomycetes, a number of new genera and species were described since the reclassification of Bensingtonia, Rhodotorula, and Sporobolomyces by Wang et al. (2015b). Two genera ${ }_{2}$ Libkindia and Yurkovia ${ }_{2}$ were proposed to accommodate new yeasts from forest soils in the Czech Republic (Mašínová et al. 2017b). The genus Heitmania was recently described to include three novel yeasts from plants in China (Liu et al. 2017). Despite being based on multi-gene analyses, the taxonomic position of these genera remained unclear and they were placed in the Microbotryomycetes as 'incertae sedis'. Wang et al. (2015b) noted that using the signal of the LSU rRNA gene alone is not sufficient to resolve the high-level phylogenetic relationships in Microbotryomycetes. Our analyses indicated two loosely placed clusters in Microbotryomycetes close to genera Curvibasidium, Pseudoleucosporidium and Sampaiozyma (Fig. 2). These weakly supported clades contained yeasts isolated from Germany (forest soil, Yurkov et al. 2016a), Portugal (plant, Inacio 2003) and Russia. A robust multi-gene analysis is required to resolve the phylogenetic relationships and taxonomic position of these yeasts. The constrained LSU rRNA gene phylogenetic analysis performed by Wang et al. (2015 b) failed to resolve placement of several psychrophilic yeasts, including Rhodotorula svalbardensis and a few undescribed species. In agreement with this observation, our results showed the unclear placement of CRUB 1733 (GenBank FJ841888) and DBVPG 10048 (GenBank KC433880) close to the filamentous fungus Camptobasidium (Fig. 2). The analysis also suggested a potential new species in this yet monotypic genus represented by an antarctic yeast, CBS 8941 (Fig. 2). Two species of Hamamotoa and one species of Colacogloea, Leucosporidium and Slooffia were described recently from soils in Europe (Yurkov et al. 2016a; Mašínová et al. 2017b). A few more novelties in genera Bannozyma (KY558342, Mašínová et al. 2017b, 2018; this study), Chrysozyma (AB552933, KX067789, Endoh et al. 2011; Wang et al. 2015b), Colacogloea (EU002850, FN428953, Kachalkin et al. 2008; Wang et al. 2015b), Fellozyma (FN868158, Wang et al. 2015b), Hamamotoa (AM039679, KU609479, AB462346, EF585181, Wang et al. 2015b; this study), Oberwinklerozyma (FN401525, Wang et al. 2015b; Yurkov et al. 2016b), Slooffia (AF444728, DQ531946, EF450537, Sampaio et al. 2011; Wang et al. 2015b) and Yurkovia (FN428970, Mašínová et al. 2017b) were reported and analysed in previous studies and await a formal description. For the first time our analysis also revealed the diversity of the genus Yunzhangia (previously known from two species only) suggesting three novel species close to Yunzhangia sonckii (Fig. 2). 
Description of Vustinia Kachalkin, Turchetti \& Yurkov, gen. nov. (MB 829115)

405

406

407

408

409

410

411

412

413

414

415

416

417

418

419

420

421

422

423

424

425

426

427

428

429

430

431

432

433

434

435

436

437

438

439

Etymology: the genus is named in honor of the Russian zymologist Dr. Michael Vustin (VKPM culture collection, State Research Institute of Genetics and Selection of Industrial Microorganisms, Russia) for his contributions to the study of soil yeasts and yeasts producing carotenoid pigments.

This monotypic genus is proposed to accommodate a new species represented by two isolates, which are phylogenetically close to the genus Krasilnikovozyma in a well-supported clade (Fig. 3).

Phylogenetic position: Fungi, Dikarya, Basidiomycota, Agaricomycotina, Tremellomycetes, Cystofilobasidiales, Mrakiaceae.

Basidiocarps absent. True hyphae and pseudohyphae not observed. Sexual reproduction not observed. Budding cells present. Budding is on the wide basis, polar with sympodial proliferation. Ballistoconidia absent. Arthroconidia absent. Urea hydrolysis and Diazonium Blue B reaction are positive. Nitrate is utilized.

Type species: Vustinia terrae Kachalkin, Turchetti \& Yurkov, sp. nov. (MB 829116)

Notes: New species of the genus Vustinia can be distinguished from known Krasilnikovozyma species by orange-colored pigmentation, which is an important character in Tremellomycetes. This is the third pigmented genus in Cystofilobasidiales after Cystofilobasidium and Phaffia. The genus Vustinia was distant from known Krasilnikovozyma species in a concatenated ITSLSU and ITS-LSU-TEF1 phylogenies (Figure 3).

\section{Description of Vustinia terrae Kachalkin, Turchetti \& Yurkov, sp. nov. (MB 829116)}

Etymology: The species epithet terrae is derived from terra (L. gen. sing. f., n., of the earth) and refers to the original substrate of isolation, namely soil.

After 1 week at $20{ }^{\circ} \mathrm{C}$ on PD, GPY and 2\% Glucose YNB agars, streak is orange, butyrous with a smooth glistening surface. Margins are smooth and entire. Cells are ellipsoidal to cylindrical 6-10 $\times 2-3 \mu \mathrm{m}$ in size, occurring singly or in pairs, and proliferating by polar budding (Figure 7 a). Lipid-like bodies can be present in cells. Pseudohyphae and true hyphae were not observed. Ballistoconidia were not observed. Teleomorph was not observed.

Glucose is not fermented. Positive growth on D-glucose, D-galactose, L-sorbose, D-ribose (weak), D-xylose, L-arabinose, D-arabinose (weak and delayed), L-rhamnose, sucrose, maltose (weak), trehalose, cellobiose, salicin (weak), arbutin (delay for some strains), melibiose (variable, delayed), lactose, raffinose (variable), melezitose (variable), soluble starch (variable, delayed), glycerol, erythritol, ribitol, xylitol (delay), D-mannitol, D-glucitol, 2-keto-D-gluconate, 5-keto-D-gluconate, D-gluconate, D-glucoronate (variable), succinate (delayed) and citrate (variable). No growth on D-glucosamine, methyl-alpha-D-glucoside, 
inulin, inositol, methanol, ethanol, galactitol, DL-lactate, L-malate and ethyl acetate.

441 Utilization of nitrogen sources: positive growth on potassium nitrate, sodium nitrite, lysine

442 and cadaverine. No growth in the presence of $10 \%$ sodium chloride, $50 \%$ D-glucose and

$4430.01 \%$ cycloheximide. Growth on vitamin-free medium is weak. Urea hydrolysis and

444 Diazonium Blue B reaction are positive. Starch-like compounds are produced. Maximum

445 growth temperature: $25^{\circ} \mathrm{C}$

446 Molecular characteristics (holotype): nucleotide sequences of ITS and LSU (D1/D2 domains)

447 rRNA, and partial sequence of TEF1 deposited in NCBI/EMBL (GenBank) under the

448 accession numbers: MH685196, MH697747 and LS992156, respectively.

449 Deposits: holotype, strain K833b isolated from mountain soil collected in June 2015 near

450 Kosh-Agach village, Republic of Altay, Russia, preserved in a metabolically inactive state in

451 the KBP collection of Department of Soil Biology, Faculty of Soil Sciences, Lomonosov

452 Moscow State University (WDCM 1173) as KBP Y-5245 ${ }^{\mathrm{T}}$. Ex-type cultures are deposited in

453 the All-Russian Collection of Microorganisms (VKM), Pushchino, Russia (VKM Y-3018),

454 the Russian National Collection of Industrial Microorganisms (VKPM), Moscow, Russia

455 (VKPM Y-4321) and the German Collection of Microorganisms and Cell Cultures (DSMZ),

456 Braunschweig, Germany (DSM 105056).

457 Strains studied: K833b (= KBP Y-5245²); paratypes: DBVPG 10597 from forest soil

458 collected in Kleiner Priol (Montiggl), South Tyrol, Alps, Italy (GenBank KU745368,

459 KU745306, MH986600) and KBP Y-5336 (= VKM Y-3019) from soil collected in a park in

460 Bishkek, Kyrgyzstan (GenBank MH697748, LS992158).

461 Notes: New species of the genus Vustinia can be distinguished from known Krasilnikovozyma

462 species by orange-colored pigmentation, positive growth on glycerol and erythritol and

463 inability to grow on D-glucosamine.

464

465

\section{Description of Udeniomyces caspiensis Kachalkin, sp. nov. (MB 829119)}

466 Etymology: The species epithet caspiensis is derived from Latin Caspius (L. adj. m., Caspian) 467 and refers to the region, where the species was isolated.

After 1 week at $20^{\circ} \mathrm{C}$ on PD, GPY and 2\% Glucose YNB agars, streak is pinkish-white, soft with a dull to semi-shiny surface. Margins are smooth and entire. Cells are ovoid to ellipsoidal, 6-11 $\times 4-5 \mu \mathrm{m}$ in size, occurring singly or in pairs, and proliferating by polar budding on a broad base (Figure $7 \mathrm{~b}$ ). Budding scars may be present. Pseudohyphae and true

472 hyphae were not observed. Ballistoconidia were not observed. Teleomorph was not observed.

473 Glucose is not fermented. Positive growth on D-glucose, D-ribose (weak), L-arabinose, D474 arabinose (weak), sucrose, maltose, trehalose, cellobiose, salicin (weak), arbutin (delay for some strains), raffinose, melezitose, glycerol, D-mannitol (weak), D-glucitol (weak), Dglucoronate (weak), D-gluconate (weak), 2-keto-D-gluconate (weak) and 5-keto-D-gluconate. No growth on D-galactose, L-sorbose, D-glucosamine, D-xylose, L-rhamnose, methyl-alphaD-glucoside, melibiose, lactose, inulin, soluble starch, ethanol, erythritol, ribitol, galactitol, inositol, DL-lactate, succinate and citrate. Utilization of nitrogen sources: positive growth on 
potassium nitrate and lysine. No growth in the presence of $10 \%$ sodium chloride and $50 \% \mathrm{D}$ glucose. Growth on vitamin-free medium is negative. Urea hydrolysis and Diazonium Blue B reaction are positive. Starch-like compounds are produced. Maximum growth temperature: 23 ${ }^{\circ} \mathrm{C}$.

Molecular characteristics (holotype): nucleotide sequences of ITS and LSU (D1/D2 domains) rRNA deposited in NCBI/EMBL (GenBank) under the accession number MH697745.

Deposits: holotype, strain K686-1b isolated from leaves of Camphorosma sp.

(Amaranthaceae) collected in May 2014 at the Djanybek Research Station of Institute of Forest Science RAS, Volgograd Oblast, Russia, preserved in a metabolically inactive state in the KBP collection of Department of Soil Biology, Faculty of Soil Sciences, Lomonosov Moscow State University (WDCM 1173) as KBP Y-5036 ${ }^{\mathrm{T}}$. Ex-type cultures are deposited in the All-Russian Collection of Microorganisms (VKM), Pushchino, Russia (VKM Y-3016), the CBS yeast collection of the Westerdijk Fungal Biodiversity Institute, Utrecht, the Netherlands (CBS 15357) and the German Collection of Microorganisms and Cell Cultures (DSMZ), Braunschweig, Germany (DSM 106747).

\section{Description of Udeniomyces orazovii Kachalkin, sp. nov. (MB 829120)}

Etymology: The species epithet orazovii (NL. gen. sing. masc. n.) is in honor of the mycologist Prof. Hodjanazar Orazov (Institute of Botany of the Academy of Sciences of Turkmenistan) for his contributions to the study of fungi in arid soils. Prof. Orazov also provided the sample from which this species was isolated.

After 1 week at $20^{\circ} \mathrm{C}$ on PD, GPY and 2\% Glucose YNB agars, streak is white, butyrous with a smooth glistening surface. Margins are undulating with some outgrowth in a medium underneath the streak. Cells are sub-globose, ellipsoidal, elongate to cylindrical, 6-15 × 3-6 $\mu \mathrm{m}$ in size, occurring singly, in pairs or chains, and proliferating by polar budding on a broad base (Figure $7 \mathrm{~d}$ ). Ballistoconidia were not observed. Pseudohyphae and short true hyphae occur. Terminal, lateral and intercalary spherical chlamydospores, 18-20 $\mu \mathrm{m}$ in size, with refractile granules from a single cell on true hyphae formed on PDA and 2\% Glucose YNB agar after 8-10 d at $20^{\circ} \mathrm{C}$ (Figure $7 \mathrm{e}, \mathrm{f}$ ). Teleomorph was not observed.

Glucose is not fermented. Positive growth on D-glucose, L-sorbose (variable), D-glucosamine (variable), D-ribose (weak), D-xylose (variable), L-arabinose, D-arabinose (weak), sucrose, maltose, trehalose, cellobiose, salicin, arbutin, lactose (variable), raffinose, melezitose, soluble starch (weak), glycerol (weak), ribitol (variable), D-glucitol, D-mannitol, inositol (weak), ethanol, 2-keto-D-gluconate, 5-keto-D-gluconate, D-glucoronate (weak), succinate (weak) and citrate (weak). No growth on D-galactose, L-rhamnose, methyl-alpha-Dglucoside, melibiose, inulin, erythritol, galactitol, D-gluconate and DL-lactate. Utilization of nitrogen sources: positive growth on potassium nitrate and lysine. No growth in the presence of $50 \%$ D-glucose. Growth on vitamin-free medium is positive. Urea hydrolysis and Diazonium Blue B reaction are positive. Starch-like compounds are produced. Maximum growth temperature: $24{ }^{\circ} \mathrm{C}$. 
Molecular characteristics (holotype): nucleotide sequences of ITS and LSU (D1/D2 domains) rRNA, and partial sequence of TEF1 deposited in NCBI/EMBL (GenBank) under the accession numbers: MH734791, MH697744 and LS998026, respectively.

523

524

525

526

527

528

529

530

531

532

533

534

535

Deposits: holotype, strain K515b isolated from leaves of Halocharis hispida (Amaranthaceae) collected in May 2013 near Babadurmaz village, Turkmenistan (approx. coordinates $37.65 \mathrm{~N}$, $59.15 \mathrm{E}$ ), preserved in a metabolically inactive state in the KBP collection of Department of Soil Biology, Faculty of Soil Sciences, Lomonosov Moscow State University (WDCM 1173) as KBP Y-4766 ${ }^{\mathrm{T}}$. Ex-type cultures are deposited in the All-Russian Collection of Microorganisms (VKM), Pushchino, Russia (VKM Y-3014), the CBS yeast collection of the Westerdijk Fungal Biodiversity Institute, Utrecht, the Netherlands (CBS xxxxx) and the German Collection of Microorganisms and Cell Cultures (DSMZ), Braunschweig, Germany (DSM 100168).

Strains studied: K515b (= KBP Y-4766 ${ }^{\mathrm{T}}$ ); paratype KBP Y-4704 (GenBank MH697743) from soil near Babadurmaz village, Turkmenistan.

\section{Description of Tausonia rosea Kachalkin, sp. nov. (MB 829122)}

Etymology: The species epithet rosea is derived from roseus (L. adj. f., pink) and refers to the culture color.

After 2 weeks at $20{ }^{\circ} \mathrm{C}$ on PD, GPY and 2\% Glucose YNB agars, streak is light pink, butyrous, with a dull and smooth surface. Margins are smooth and entire. Cells are globose, sub-globose and ovoid, 7-8 × 4.5-7 $\mu \mathrm{m}$ in size, occurring singly or in pairs, and proliferating by polar budding on a broad base (Figure $7 \mathrm{c}$ ). Budding scars may be present. Spherical chlamydospore-like cells, 8-10 $\mu \mathrm{m}$ in size, may be present in older culture. Pseudohyphae and true hyphae were not observed. Ballistoconidia were not observed. Teleomorph was not observed.

Glucose is not fermented. Positive growth on D-glucose, L-arabinose, D-arabinose, sucrose, maltose, trehalose, cellobiose (weak), salicin, arbutin, raffinose, melezitose, soluble starch (weak), glycerol (weak), ribitol (weak), D-glucitol, D-mannitol, 2-keto-D-gluconate, 5-ketoD-gluconate (weak), D-glucoronate (weak) and ethanol (weak). No growth on D-galactose, Lsorbose, D-glucosamine, D-ribose, D-xylose, L-rhamnose, methyl-alpha-D-glucoside, melibiose, lactose, inulin, erythritol, galactitol, inositol, D-gluconate, DL-lactate, succinate and citrate. Utilization of nitrogen sources: positive growth on potassium nitrate and lysine. No growth in the presence of $10 \%$ sodium chloride and 50\% D-glucose. Growth on vitaminfree medium is positive. Urea hydrolysis and Diazonium Blue B reaction are positive. Starchlike compounds are produced. Maximum growth temperature: $23{ }^{\circ} \mathrm{C}$.

Molecular characteristics (holotype): nucleotide sequences of ITS and LSU (D1/D2 domains) rRNA, and partial sequence of TEF1 deposited in NCBI/EMBL (GenBank) under the accession numbers: LN871177 and LS998027, respectively.

Deposits: holotype, strain K744-1b isolated from leaves of Salicornia sp. (Amaranthaceae) collected in August 2014 on the shore of lake Elton, Volgograd Oblast, Russia, (approx. 
coordinates $49.15 \mathrm{~N}, 46.68 \mathrm{E}$ ), preserved in a metabolically inactive state in the KBP collection of Department of Soil Biology, Faculty of Soil Sciences, Lomonosov Moscow State University (WDCM 1173) as KBP Y-4584 ${ }^{\mathrm{T}}$. Ex-type cultures are deposited in the AllRussian Collection of Microorganisms (VKM), Pushchino, Russia (VKM Y-3007), the CBS yeast collection of the Westerdijk Fungal Biodiversity Institute, Utrecht, the Netherlands (CBS xxxxx) and the German Collection of Microorganisms and Cell Cultures (DSMZ), Braunschweig, Germany (DSM 100302).

\section{Description of Itersonilia diksonensis Kachalkin, sp. nov. (MB 829123)}

569

570

571

Etymology: The species epithet diksonensis (NL. gen. sing. masc. n.) is derived from Dikson, the urban-type settlement on Russia's Arctic Ocean coast and refers to the region where the species was isolated.

After 2 weeks at $20^{\circ} \mathrm{C}$ on GPY and 2\% Glucose YNB agars, streak is salmon-pink, butyrous, with a glossy wrinkled surface. Margins are crenulate and fringed with tiny colonies resulting from discharged ballistoconidia. Cells are ellipsoidal, sub-globose to ovoid, 6-9 $64.5-6 \mu \mathrm{m}$ in size, occurring singly or in pairs, and proliferating by polar and lateral budding_Figure 7 g). Ballistoconidia are lunate, 5.5-6 × 3.5-4 $\mu \mathrm{m}$ size, formed abundantly on cells with 1-2 occasionally sympodially branching sterigmata_(Figure $7 \mathrm{~h}$ ). After 2 weeks at $20^{\circ} \mathrm{C}$ on PDA, streak is light salmon-pink, mucoid, raised with a smooth and glossy surface. Margin is entire. Cells are ovoid, ellipsoidal or elongate, 6-12 × 5-6 $\mu \mathrm{m}$ in size, occur singly, in pairs or chains, and proliferating by polar budding. Rare ballistoconidia formed at the end of 10-30 $\mu \mathrm{m}$ long sterigmata. Pseudohyphae and true hyphae were not observed on any media. Teleomorph was not observed.

Glucose is not fermented. Positive growth on D-glucose, L-sorbose, D-ribose, D-xylose, Larabinose, D-arabinose, L-rhamnose, sucrose, trehalose, cellobiose, salicin, arbutin, raffinose, melezitose (weak), soluble starch (weak), glycerol, ribitol, D-glucitol, D-mannitol, 5-keto-Dgluconate (weak), D-gluconate, D-glucoronate (weak), DL-lactate (weak), succinate, citrate and ethanol. No growth on D-galactose, D-glucosamine, maltose, methyl-alpha-D-glucoside, melibiose, lactose, inulin, erythritol, galactitol and inositol. Utilization of nitrogen sources: positive growth on potassium nitrate and lysine. No growth in the presence of 50\% D-glucose. Growth on vitamin-free medium is positive. Urea hydrolysis and Diazonium Blue B reaction are positive. Starch-like compounds are not produced. Maximum growth temperature: $36^{\circ} \mathrm{C}$.

Molecular characteristics (holotype): nucleotide sequences of ITS and LSU (D1/D2 domains) rRNA, and partial sequence of TEF1 deposited in NCBI/EMBL (GenBank) under the accession numbers: MH697741, MH734790 and LS998025, respectively.

Deposits: holotype, strain K343b isolated from a mixed sample of leaves of flowering arctic plants collected in July 2012 near the settlement Dikson, Taymyr peninsular, Krasnoyarsk Krai, Russia, preserved in a metabolically inactive state in the KBP collection of Department of Soil Biology, Faculty of Soil Sciences, Lomonosov Moscow State University (WDCM 1173) as KBP Y- $4765^{\mathrm{T}}$. Ex-type cultures are deposited in the All-Russian Collection of 
Microorganisms (VKM), Pushchino, Russia (VKM Y-3013), the Russian National Collection of Industrial Microorganisms (VKPM), Moscow, Russia (VKPM Y-4017), the CBS yeast collection of the Westerdijk Fungal Biodiversity Institute, Utrecht, the Netherlands (CBS xxxxx) and the German Collection of Microorganisms and Cell Cultures (DSMZ), Braunschweig, Germany (DSM 100169).

\section{Description of Krasilnikovozyma fibulata Glushakova \& Kachalkin sp. nov. (MB 829124)}

Etymology: The species epithet fibulata is derived from fibula (L. adj. f., with clamp) and refers to the presence of hyphae with clamp connections.

After 2 weeks at $20{ }^{\circ} \mathrm{C}$ on PD, GPY and 2\% Glucose YNB agars, streak is cream colored, butyrous with a smooth glistening surface and hyphe produced at the margin. Cells are fusiform, $6-10 \times 2-3 \mu \mathrm{m}$ in size, and occurring singly or in pairs, and proliferating by polar budding (Figure 7 i). Ballistoconidia were not observed. Pseudohyphae and true hyphae with clamp connections occur. Spherical teliospores, 15-16 $\mu \mathrm{m}$ in size, are produced laterally or terminally (Figure $7 \mathrm{j}, \mathrm{k}$ ). No teliospore germination was observed and basidia morphology remains unknown.

Glucose is not fermented. Positive growth on D-glucose, D-galactose, L-sorbose, Dglucosamine, D-xylose, L-rhamnose, sucrose, trehalose, cellobiose, salicin (weak), arbutin, lactose, ethanol (weak), ribitol (weak), D-glucitol, 2-keto-D-gluconate, 5-keto-D-gluconate, citrate (weak) succinate (weak) and ethanol. No growth on L-sorbose, D-ribose, L-arabinose, D-arabinose, maltose, methyl-alpha-D-glucoside, melibiose, raffinose, melezitose, inulin, soluble starch, glycerol, erythritol, D-mannitol, galactitol, inositol, D-gluconate, Dglucoronate and DL-lactate. Utilization of nitrogen sources: positive growth on potassium nitrate and lysine. No growth in the presence of 50\% D-glucose. Growth on vitamin-free medium is positive. Urea hydrolysis and Diazonium Blue B reaction are positive. Starch-like compounds are produced. Maximum growth temperature: $24{ }^{\circ} \mathrm{C}$.

Molecular characteristics (holotype): nucleotide sequences of ITS and LSU (D1/D2 domains) rRNA, and partial sequence of TEF1 deposited in NCBI/EMBL (GenBank) under the accession numbers: MH685197, MH697754 and LS992159, respectively.

Deposits: holotype, strain A528 isolated from roots of Impatiens parviflora (Balsaminaceae), in September 2010 in the Losiny Ostrov (Rus. Elk Island) National Park, Moscow, Russia, preserved in a metabolically inactive state in the KBP collection of Department of Soil Biology, Faculty of Soil Sciences, Lomonosov Moscow State University (WDCM 1173) as KBP Y-5098 ${ }^{\mathrm{T}}$. Ex-type cultures are deposited in the All-Russian Collection of Microorganisms (VKM), Pushchino, Russia (VKM Y-3017), the Russian National Collection of Industrial Microorganisms (VKPM), Moscow, Russia (VKPM Y-3827), the CBS yeast collection of the Westerdijk Fungal Biodiversity Institute, Utrecht, the Netherlands (CBS xxxxx) and the German Collection of Microorganisms and Cell Cultures (DSMZ), Braunschweig, Germany (DSM 105054). 
642 Basionym: Mrakia curviuscula Babeva, Lisichkina, Reshetova \& Danilevich MB 529873

643 Holotype: KBP Y-3618 isolated from moss Bryum sp., dry white moss-pine forest, Oka

644

645

646

647

648

649

650

651

652

653

654

655

656

657

658

659

660

661

662

663

664

665

666

667

668 Nature Reserve, Ryazan Oblast, Russia, preserved in a metabolically inactive state (dried) in the KBP collection of Department of Soil Biology, Faculty of Soil Sciences, Lomonosov Moscow State University (WDCM 1173).

Ex-type cultures are deposited in the All-Russian Collection of Microorganisms (VKM), Pushchino, Russia (VKM Y-2953) and the Portuguese Yeast Culture Collection (PYCC), Caparica, Portugal (PYCC 5836).

Molecular characteristics (holotype): nucleotide sequences of ITS and LSU (D1/D2 domains) rRNA deposited in NCBI/EMBL (GenBank) under the accession numbers: MK244628 (VKM Y-2953), and MF372124 and MF372143 (PYCC 5836), respectively.

Ecology: The species is widespread. It was found in soil (Japan, Canada, USA, Denmark; GenBank AB462342, KM384610, MH655679, MG914803), in mushroom (Taiwan; GenBank FJ873516), pine forest litter (Russia; GenBank MK244629, MK244630), rotten wood (Argentina), Sphagnum moss (Russia), and orchid flower (North America; GenBank EU218880) (e.g. Taylor and McCormick 2008, Glushakova et al. 2015, Sylvester et al. 2015).

\section{Tremellomycetes, Tremellales}

Description of Hannaella taiwanensis (F.L. Lee \& C.H. Huang) Yurkov comb. nov. (MB 829114)

Basionym: Cryptococcus taiwanensis F.L. Lee \& C.H. Huang , Fungal Science 26 (1): 61 (2011); MB 560182

Note: The species was overlooked in the analysis by Liu et al. (2015). Sequences D1/D2 domains of LSU (HQ591443) of the type strain BCRC 23252 show 97\% similarity to Hannaella zeae, H. kunmingensis, H. siamensis, and 96\% similarity to the type species of the genus $H$. sinensis (Fig. S1).

Validation of Tremella basidiomaticola X.Z. Liu \& F.Y. Bai, MycoKeys 47:80 (2018); MB $\underline{827184}$

Zhao et al. (2018) described four new Tremella species from China. Type material of the three sexual species (Tremella cheejenii, T. erythrina, and T. salmonea) was deposited in the Herbarium of the Chinese Academy of Sciences (acronym HMAS). The species Tremella basidiomaticola is known from its asexual (yeast) state, and the holotype (a strain) was deposited in the China General Microbiological Culture Collection Center. The description of Tremella basidiomaticola does not conform with the ICN Shenzhen Code. According to the Article 40.8 of the ICN Shenzhen Code, for the name of a new species or infraspecific taxon 
published on or after 1 January 2019 of which the type is a culture, the protologue must include a statement that the culture is preserved in a metabolically inactive state. This requirement was not fulfilled in the original description by Zhao et al. (2018). Therefore, the description is validated here in accordance with the Article 9.2 of the ICN Shenzhen Code.

Holotype, strain CGMCC $2.5724^{\mathrm{T}}$ isolated from a basidioma of Tremella fuciformis collected in July 2017 by X.Z. Liu in Gutian county, Ningde city, Fujian Province, China, preserved in a metabolically inactive state at the China General Microbiological Culture Collection Center, Beijing, China. Ex-holotype culture is deposited in the CBS yeast collection of the Westerdijk Fungal Biodiversity Institute, Utrecht, the Netherlands (CBS 15261).

\section{Comments to the genus Gelidatrema}

The description of the second species of the genus Gelidatrema, Gelidatrema psychrophila, was published simultaneously with the preparation of the present manuscript (Tsuji et al. 2018). This species was described based on two isolates from a microbial mat in the Canadian High Arctic. Another culture of Gelidatrema psychrophila (DBVPG 5459, GenBank KC433781) was isolated from a snow sample in Italian Alps (Turchetti et al. 2013). This culture has not been considered in the description by Tsuji et al. (2018). We compared physiological profiles of Gelidatrema psychrophila from the original description (Tsuji et al. 2017) with those of the strain DBVPG 5459 (this study). In contrast to results published by (Tsuji et al. 2018), the strain G. psychrophila DBVPG 5459 grew on D-gluconate but not on D-glucoronate, N-acetyl-D-glucosamine, and succinate. Assimilation of glycerol and ethanol was weak or negative (Tsuji et al. 2018; this study). Similarly to results obtained for the type species of the genus, G. spencermartinsiae, and in contrast to results published by Tsuji et al. (2018), the strain DBVPG 5459 did not assimilate potassium nitrate and sodium nitrite.

The two species of the genus Gelidatrema (G. spencermartinsiae and G. psychrophila) are polytrophic utilizing a total of 22 carbon sources, namely arbutin, cellobiose, D-arabinose, Dgalactose, D-xylose, D-glucose, galactitol, L-arabinose, L-rhamnose, D-ribose, D-mannitol, lactose, maltose, melezitose, melibiose, myo-inositol, raffinose, ribitol, salicin, sorbitol, trehalose, and xylitol. Both species do not grow on citrate, erythritol, ethanol, ethyl-acetate, glycerol, hexadecane, inulin, lactate, L-sorbose and methanol. Amino acids lysine and cadaverine are utilised as a source of nitrogen, but no growth occurs on potassium nitrate and sodium nitrite. Taking together, the results of the present study and descriptions of $G$. spencermartinsiae and G. psychrophila (de Garcia et al. 2010; Tsuji et al. 2018), the two species can be only distinguished on the basis of utilisation of D-ribose, sucrose, Dglucosamine and ethylamine.

\section{Description of Kwoniella fici Turchetti \& Buzzini sp. nov. (MB 829127)}

Etymology: The species epithet fici is derived from ficus (L. gen. sing. n. n., of fig tree) and refers to the substrate of isolation. 
After 1 week at $25^{\circ} \mathrm{C}$ on MEA, PDA and GPYA, streak culture is greyish-white (MEA, PDA) to cream-colored (GPYA), viscous to butyrous with a dull smooth surface. Margins are smooth and entire and the profile is flat. Cells are spherical to globose, 5-5.5 $\mu \mathrm{m}$, occurring singly or in pairs, and proliferating by multilateral budding. Pseudohyphae and true hyphae were not observed. Ballistoconidia were not observed. Teleomorph was not observed.

Glucose is not fermented. Positive growth on D-glucose, D-galactose (weak, delayed), Dribose, D-xylose, L-arabinose, D-arabinose, L-rhamnose, sucrose, maltose, trehalose, methylalpha-D-glucoside (weak), cellobiose, salicin (weak, delayed), lactose, raffinose, melezitose, glycerol, erythritol, ribitol, xylitol, D-glucitol, D-mannitol, galactitol, myo-inositol, gluconodelta-lactone, D-gluconate, succinate and ethanol. No growth on L-sorbose, arbutin, melibiose, D-glucoronate, D-galacturonate, DL-lactate, citrate, methanol, L-malate, hexadecane, N-acetyl-D-glucosamine, and ethyl acetate. Utilisation of nitrogen sources: positive growth on potassium nitrate, sodium nitrite, ethylamine, lysine and cadaverine. No growth in the presence of $0.01 \%$ cycloheximide. Growth in the presence of $10 \% \mathrm{NaCl}$ and 50 $\%$ glucose is negative. Growth on vitamin-free medium is weak. Urea hydrolysis and Diazonium Blue B reaction are positive. Starch-like compounds are abundantly produced. Maximum growth temperature: $30{ }^{\circ} \mathrm{C}$.

Molecular characteristics (holotype): nucleotide sequences of ITS and LSU (D1/D2 domains) rRNA deposited in NCBI/EMBL (GenBank) under the accession numbers: MK070336 and MK070318, respectively.

Deposits: holotype, strain AH-10 isolated from a fruit of the common fig (Ficus carica) collected in September 2011 in Umbria, Italy, preserved in a metabolically inactive state in the Industrial Yeasts Collection DBVPG, Department of Agricultural, Food and Environmental Sciences, University of Perugia, Perugia, Italy as DBVPG $10122^{\mathrm{T}}$.

\section{Tremellomycetes, Filobasidiales}

\section{Description of Heterocephalacria fruticeti f.a. Carvalho, Roehl, Yurkov \& Sampaio sp. nov. (MB 829128)}

Etymology: The species epithet fruticeti is derived from fruticetum (L. gen. sing. n. n., of a thicket) and refers to the substrate of isolation, which was a dense maqui scrubland.

After 1 week at $25^{\circ} \mathrm{C}$ on GPY agar and PDA, streak culture is whitish to cream-coloured, mucoid with a glistering smooth surface. Margins are smooth and entire and the profile is flat. Cells are oval to ellipsoidal 3-5 $\times 5-7 \mu \mathrm{m}$ in size, occurring singly or in pairs, and proliferating by polar and multilateral budding. Pseudohyphae and true hyphae were not observed. Ballistoconidia were not observed. Teleomorph was not observed.

Glucose is not fermented. Positive growth on D-glucose, D-galactose, L-sorbose, L-arabinose, D-arabinose, L-rhamnose, sucrose, maltose, trehalose, methyl-alpha-D-glucoside, cellobiose, salicin, lactose, melezitose, inulin (weak), soluble starch (delayed), ribitol, xylitol, D-glucitol, D-mannitol, inositol, D-glucuronate, succinate, citrate, L-malate, L-tartrate, methanol (weak) and ethanol. No growth on D-glucosamine, D-ribose, D-xylose, melibiose, raffinose, glycerol, 
erythritol, galactitol, glucono-delta-lactone, D-gluconate and DL-lactate. Utilisation of nitrogen sources: positive growth on potassium nitrate, sodium nitrite, lysine and cadaverine. Growth in the presence of $0.01 \%$ and $0.1 \%$ cycloheximide is positive. Growth in the presence of $10 \% \mathrm{NaCl}$ is negative. Growth on vitamin-free medium is negative. Urea hydrolysis and Diazonium Blue B reaction are positive. Starch-like compounds are produced. Maximum growth temperature: $25^{\circ} \mathrm{C}$.

Molecular characteristics (holotype): nucleotide sequences of ITS and LSU (D1/D2 domains) rRNA deposited in NCBI/EMBL (GenBank) under the accession numbers: MK307720 and KT314192, respectively.

Deposits: holotype, strain OR 402 isolated from dry scrubland soil collected in the Arrábida Natural Park, Serra da Arrábida, Portugal, preserved in a metabolically inactive state in the Portuguese Yeast Culture Collection, Caparica, Portugal as PYCC 8314 ${ }^{\mathrm{T}}$.

\section{Description of Heterocephalacria gelida f.a. Turchetti \& Kachalkin sp. nov. (MB 829129)}

Etymology: The species epithet gelida is derived from gelidus (L. f. adj., frosty) and refers to the climatic conditions of localities, from which the species was isolated.

After 1 week at $25^{\circ} \mathrm{C}$ on GPY agar and PDA, streak culture is whitish to cream-colored, mucoid and viscous with a glistering smooth surface. Margins are smooth and entire, and the profile is flat. Cells are sub-globose, oval to ellipsoidal 3-5 $\times 7-9 \mu \mathrm{m}$ in size, occurring singly, in pairs or short chains, and proliferating by polar and multilateral budding. Pseudohyphae and true hyphae were not observed. Ballistoconidia were not observed. Sediment is produced when the strain grows in ME and GPY broth at $25^{\circ} \mathrm{C}$ after 7 days. After 14 days, a superficial ring is also present. Teleomorph not observed.

Glucose is not fermented. Positive growth on glucose, D-galactose, L-sorbose, Dglucosamine, D-ribose (weak), D-xylose, L-arabinose, D-arabinose (weak), L-rhamnose, sucrose, maltose, trehalose, methyl alpha-methyl-D-glucoside, cellobiose, salicin, arbutin, melibiose (weak), lactose, raffinose, melezitose, ribitol, xylitol, D-glucitol, D-mannitol, galactitol, myo-inositol, glucono-delta-lactone, D-gluconate, and D-galacturonate (weak). No growth on glycerol, erythritol, D-glucoronate, DL-lactate, succinate, citrate, ethanol, methanol, L-malic acid, hexadecane, $\mathrm{N}$-acetyl-D-glucosamine and ethyl acetate. Utilisation of nitrogen sources: positive growth on potassium nitrate, sodium nitrite, ethylamine and lysine. Growth in the presence of $0.01 \%$ is weak; no growth in the presence of $0.1 \%$ cycloheximide. Growth in the presence of $10 \% \mathrm{NaCl}$ and $50 \%$ glucose is negative. Growth on vitamin-free medium is negative. Urea hydrolysis and Diazonium Blue B reaction are positive. Starch-like compounds are produced. Maximum growth temperature: $25^{\circ} \mathrm{C}$.

Molecular characteristics (holotype): nucleotide sequences of ITS and LSU (D1/D2 domains) rRNA deposited in NCBI/EMBL (GenBank) under the accession numbers: KC455903 and KC433839, respectively.

Deposits: holotype, strain 20.9 LB.6 isolated from supraglacial sediments collected in July 2009 in Miage glacier, Mont Blanc massif, Alps, Italy (45.783333N, 6.866667E), preserved 
in a metabolically inactive state in the Industrial Yeasts Collection DBVPG, Department of Agricultural, Food and Environmental Sciences, University of Perugia, Perugia, Italy as DBVPG $5868^{\mathrm{T}}$.

800

801

802

803

804

805

806

807

808

809

B10

811

812

813

814

815

816

817

818

819

820

821

822

823

824

825

826

827

828

829

830

831

832

833

834

835

Strains studied: DBVPG 5868 ${ }^{\mathrm{T}}$, paratype KBP Y-5466 from leaves of Dryas punctata (Rosaceae) collected near the settlement Dikson, Taymyr peninsular, Krasnoyarsk Krai, Russia.

\section{Description of Heterocephalacria hypogea f.a. Carvalho, Roehl, Yurkov \& Sampaio sp. nov. (MB 829130)}

Etymology: The species epithet hypogea is derived from hypogeus (L. f. adj., underground) and refers to the substrate of isolation.

After 1 week at $25^{\circ} \mathrm{C}$ on GPY agar and PDA, streak culture is whitish to cream-coloured, mucoid with a glistering smooth surface. Margins are smooth and entire and the profile is flat. Cells are oval to ellipsoidal 3-5 × 5-7 $\mu \mathrm{m}$ in size, occurring singly or in pairs, and proliferating by polar and multilateral budding. Pseudohyphae and true hyphae were not observed. Ballistoconidia were not observed. Teleomorph was not observed.

Glucose is not fermented. Positive growth on D-glucose, D-galactose, L-sorbose, Dglucosamine, L-arabinose, D-arabinose, L-rhamnose, sucrose, maltose, trehalose, methylalpha-D-glucoside, cellobiose, salicin, melibiose (weak), lactose, raffinose, melezitose, inulin (weak), soluble starch (weak), ribitol, xylitol, D-glucitol, D-mannitol, galactitol, inositol, Dglucuronate, succinate, citrate, L-malate, L-tartrate, methanol (weak) and ethanol (weak). No growth on D-ribose, D-xylose, glycerol, erythritol, glucono-delta-lactone, D-gluconate and DL-lactate. Utilisation of nitrogen sources: positive growth on potassium nitrate, sodium nitrite, lysine and cadaverine. Growth in the presence of $0.01 \%$ and $0.1 \%$ cycloheximide is positive. Growth in the presence of $10 \% \mathrm{NaCl}$ is negative. Growth on vitamin-free medium is negative. Urea hydrolysis and Diazonium Blue B reaction are positive. Starch-like compounds are produced. Maximum growth temperature: $25^{\circ} \mathrm{C}$.

Molecular characteristics (holotype): nucleotide sequences of ITS and LSU (D1/D2 domains) rRNA deposited in NCBI/EMBL (GenBank) under the accession numbers: MK307721 and KT253539, respectively.

Deposits: holotype, strain OR 262 isolated from semihumid chaparral forest soil collected in the Arrábida Natural Park, Serra da Arrábida, Portugal, preserved in a metabolically inactive state in the Portuguese Yeast Culture Collection, Caparica, Portugal as PYCC 6805 ${ }^{\mathrm{T}}$.

\section{Description of Heterocephalacria Iusitanica f.a. Inacio, Carvalho, Roehl, Yurkov \&} Sampaio sp. nov. (MB 829131)

Etymology: The species epithet lusitanica (L. f. adj.) is derived from the name of the ancient Roman province Lusitania, which included the territory of modern Portugal. The epithet refers to the locality, where the species was isolated. 
After 1 week at $25^{\circ} \mathrm{C}$ on GPY agar and PDA, streak culture is whitish to cream-coloured, mucoid with a glistering smooth surface. Margins are smooth and entire and the profile is flat.

$\beta 38$ Cells are oval to ellipsoidal 3-5 × 5-7 $\mu \mathrm{m}$ in size, occurring singly or in pairs, and proliferating by polar and multilateral budding. Pseudohyphae and true hyphae were not observed. Ballistoconidia were not observed. Teleomorph was not observed.

Glucose is not fermented. Positive growth on D-glucose, D-galactose, L-sorbose, Dglucosamine, D-ribose (variable), D-xylose (variable), L-arabinose, D-arabinose, L-rhamnose, sucrose, maltose, trehalose, methyl-alpha-D-glucoside, cellobiose, salicin, melibiose, lactose, raffinose (delayed), melezitose, inulin (variable), soluble starch (variable, delayed), ribitol, xylitol, D-glucitol, D-mannitol, inositol, glucono-delta-lactone, D-gluconate, D-glucuronate, succinate (variable), citrate, L-malate, L-tartrate, saccharate and galactarate. No growth on glycerol, erythritol, DL-lactate, methanol, ethanol, D-tartrate and m-tartrate. Growth on lowweight aromatic compounds is negative. Utilisation of nitrogen sources: positive growth on potassium nitrate, sodium nitrite, ethylamine (variable), lysine and cadaverine. Growth in the presence of $0.01 \%$ and $0.1 \%$ cycloheximide is positive. Growth in the presence of $10 \% \mathrm{NaCl}$ is negative. Growth on vitamin-free medium is negative. Urea hydrolysis and Diazonium Blue $\mathrm{B}$ reaction are positive. Starch-like compounds are produced. Maximum growth temperature: $25{ }^{\circ} \mathrm{C}$.

Molecular characteristics (holotype): nucleotide sequences of ITS and LSU (D1/D2 domains) rRNA deposited in NCBI/EMBL (GenBank) under the accession numbers: MK307716 and EU002809, respectively.

Deposits: holotype, strain 2MV5 isolated from fruits of the strawberry tree (Arbutus unedo) collected in the Arrábida Natural Park, Serra da Arrábida, Portugal, preserved in a metabolically inactive state in the Portuguese Yeast Culture Collection, Caparica, Portugal as PYCC 6104 ${ }^{\mathrm{T}}$. Ex-type culture is deposited in the CBS yeast collection of the Westerdijk Fungal Biodiversity Institute, Utrecht, the Netherlands (CBS 10185).

Strains studied: 2MV5 (= CBS 10185), paratypes 4ExS4 (= CBS 10186) from an oak exudate, OR 78 (= PYCC 8313) from soil under humid sclerophyll forest. All known strains were isolated from Nature Park of Arrábida on northern and southern slopes of the Serra da Arrábida mountain range from plants and soil (Inacio 2003; Yurkov et al. 2016).

\section{Comments to the genus Heterocephalacria}

The genus Heterocephalacria proposed by Liu et al. (2015) comprises two teleomorphic mycoparasites $H$. bachmannii and $H$. physciacearum and asexual $H$. arrabidensis, which is known from yeast state only. Although no teleomorph was discovered for newly described $H$. gelida and $H$. lusitanica, a mycoparasitic lifestyle cannot be excluded. No culture is available for $H$. bachmannii and $H$. physciacearum. Physiological profiles are only available for asexual species, which can be distinguished based on assimilation of ethanol, glycerol, DLlactate, succinate, citrate, D-glucoronate, L-malate, nitrate and lysine. However, closely related $H$. gelida and $H$. lusitanica only differ in growth on succinate, citrate, D-glucoronate 
and L-malate. $H$. hypogea can be distinguished from the closely related $H$. arrabidensis in assimilation of D-xylose, D-ribose, methanol, glycerol, DL-lactate, D-glucoronate, nitrate, ethylamine, and lysine.

\section{Description of Piskurozyma arborea Yurkov, Kachalkin, Mašínová \& Baldrian sp. nov.} (MB 829132)

Etymology: The species epithet arborea refers to the habitat of isolation.

After 1 week at $25^{\circ} \mathrm{C}$ on GPY agar and PDA, streak culture is white to cream-coloured, butyrous with a glistering smooth surface. Margins are smooth and entire. Cells are ovoid, cylindrical or somewhat sausage-shaped, 6-7 × 2-4 $\mu \mathrm{m}$. Budding is polar or occurs, in the case of lateral budding, near the poles of the cells. Pseudohyphae and true hyphae were not observed. Ballistoconidia were not observed. Teleomorph was not observed.

Glucose is not fermented. Positive growth on D-glucose, D-galactose, L-sorbose, D-ribose (variable), D-xylose, L-Arabinose, D-Arabinose, L-rhamnose, sucrose, maltose, trehalose, cellobiose, salicin (variable), arbutin, melibiose, lactose, raffinose, melezitose, inulin, soluble starch, glycerol (variable), ribitol, xylitol, arabitol, D-glucitol, D-mannitol, galactitol, inositol, 2-Keto-D-Gluconate, 5-Keto-D-Gluconate, D-gluconate, D-glucuronate, DL-lactate, succinate, citrate and L-malate. No growth on D-glucosamine and erythritol. Utilization of nitrogen sources: positive growth on potassium nitrate and lysine. Growth in the presence of $10 \% \mathrm{NaCl}$ and $50 \%$ glucose is negative. Growth on vitamin-free medium is positive. Urea hydrolysis and Diazonium Blue B reaction are positive. Starch-like compounds are not produced. Maximum growth temperature: $25^{\circ} \mathrm{C}$.

Molecular characteristics (holotype): nucleotide sequences of ITS and LSU (D1/D2 domains) rRNA deposited in NCBI/EMBL (GenBank) under the accession number KY558349.

Deposits: holotype, strain KT168 isolated from spruce litter collected in Kladeruby nad Oslavou, Třebíč District, Vysočina Region, Czech Republic, preserved in a metabolically inactive state in the German Collection of Microorganisms and Cell Cultures (DSMZ), Braunschweig, Germany as DSM $103202^{\mathrm{T}}$.

Strains studied: DSM 103202 , paratype KBP Y-4682 (= VKM Y-3011) from Sphagnum moss collected in Moscow Region, Russia.

\section{Description of Piskurozyma silvicultrix Turchetti, Mašínová, Baldrian \& Yurkov sp. nov. (MB 829133)}

Etymology: The species epithet silvicultrix (L. f. adj., inhabiting forest) refers to the habitat of isolation.

After 1 week at $25^{\circ} \mathrm{C}$ on GPY agar and PDA, streak culture is white to cream-colored, mucoid with a glistering smooth surface. Margins are smooth and entire and the profile is flat. Cells are globose 7-7.5 $\mu \mathrm{m}$ in diameter or ovoid 6-7 $\times 2-4 \mu \mathrm{m}$, occurring singly, in pairs or 
short chains, and proliferating by polar budding. Pseudohyphae and true hyphae were not observed. Ballistoconidia were not observed. Teleomorph was not observed.

Glucose is not fermented. Positive growth on D-glucose, D-galactose, L-sorbose, D-xylose, L-arabinose, sucrose, maltose, trehalose, methyl-alpha-D-glucoside, arbutin, melezitose, soluble starch, erythritol, D-glucitol, D-mannitol, myo-inositol, glucono-delta-lactone (delayed), D-gluconate (delayed), D-glucoronate (delayed), D-galacturonate (delayed), DLlactate (delayed) and succinate. No growth on D-ribose, D-arabinose, L-rhamnose, cellobiose, salicin, melibiose, lactose, raffinose, glycerol, ribitol, xylitol, galactitol, citrate, ethanol, methanol, L-malate, hexadecane, $\mathrm{N}$-acetyl-D-glucosamine and ethyl acetate. Utilisation of nitrogen sources: positive growth on ethylamine; no growth on potassium nitrate, sodium nitrite, L-lysine, and cadaverine. Growth in the presence of $0.01 \%$ and $0.1 \%$ cycloheximide is positive. Growth in the presence of $10 \% \mathrm{NaCl}$ and $50 \%$ glucose is negative. Growth on vitamin-free medium is negative. Urea hydrolysis and Diazonium Blue B reaction are positive. Starch-like compounds are produced. Maximum growth temperature: $25^{\circ} \mathrm{C}$.

Molecular characteristics (type strain): nucleotide sequences of ITS and LSU (D1/D2 domains) rRNA deposited in NCBI/EMBL (GenBank) under the accession numbers: KU745333 and KU745299, respectively.

Deposits: holotype, strain 20.14 A S4 2R isolated from forest soil collected in June 2014 in Kleiner Priol (Montiggl), South Tyrol, Alps, Italy, preserved in a metabolically inactive state in the Industrial Yeasts Collection DBVPG, Department of Agricultural, Food and Environmental Sciences, University of Perugia, Perugia, Italy as DBVPG $10557^{\mathrm{T}}$.

Strains studied: DBVPG 10557 , paratypes DSM 103194 and DSM 103201 (GenBank KY558341 and KY558348, respectively) from beech litter collected in Czech Republic (Mašínová et al. 2017b).

\section{Description of Piskurozyma stramentorum Yurkov, Mašínová \& Baldrian sp. nov. (MB}

\section{4)}

Etymology: The species epithet stramentorum is derived from stramentum (L. gen. plu. n. n., litter) and refers to the substrate of isolation.

943 After 1 week at $25{ }^{\circ} \mathrm{C}$ on GPY agar and PDA, streak culture is white to cream-coloured, 944 butyrous with a glistering smooth surface. Margins are smooth and entire. Cells are ovoid, cylindrical or somewhat sausage-shaped, 6-7 × 2-4 $\mu \mathrm{m}$. Budding is polar or occurs, in the case of lateral budding, near the poles of the cells. Pseudohyphae and true hyphae were not observed. Ballistoconidia were not observed. Teleomorph was not observed.

948 Glucose is not fermented. Positive growth on D-glucose, D-galactose, L-sorbose, D949 glucosamine, D-ribose, D-xylose, L-arabinose, D-arabinose, L-rhamnose, sucrose, maltose, 950 D-trehalose, methyl-alpha-D-glucoside (weak), cellobiose, melibiose, lactose, raffinose, 951 melezitose, ribitol, D-glucitol, D-mannitol (weak), D-sorbitol (weak), galactitol, inositol, D952 Gluconate, L-malate (weak) and arbutin. No growth on salicin, inulin, glycerol, erythritol, 
potassium nitrate, ethylamine and L-lysine. Growth on vitamin-free medium is negative. Urea hydrolysis and Diazonium Blue B reaction are positive. Starch-like compounds are produced. Maximum growth temperature: $25{ }^{\circ} \mathrm{C}$.

Molecular characteristics (type strain): nucleotide sequences of ITS and LSU (D1/D2 domains) rRNA deposited in NCBI/EMBL (GenBank) under the accession number KY558344.

Deposits: holotype, strain KT146 isolated from spruce litter collected in Kladeruby nad Oslavou, Třebíč District, Vysočina Region, Czech Republic, preserved in a metabolically inactive state in the German Collection of Microorganisms and Cell Cultures (DSMZ), Braunschweig, Germany as DSM $103197^{\mathrm{T}}$.

\section{Description of Naganishia nivalis Turchetti \& Buzzini sp. nov. (MB 829135)}

Etymology: The species epithet nivalis (L. f. adj., snowy) refers to the substrate of isolation. After 1 week at $25^{\circ} \mathrm{C}$ on MEA, PDA and GPYA, streak culture is white to cream-colored (becoming brownish upon aging), viscous to butyrous with a dull wrinkled surface. Margins are smooth and entire and the profile is raised. Cells are spherical to ovoid 2.5-3 $\times 2-2.5 \mu \mathrm{m}$ in size, occurring singly or in pairs, and proliferating by polar budding. Pseudohyphae and true hyphae were not observed. Ballistoconidia were not observed. Sediment is produced when the strain grows in ME and GPY broth at $25^{\circ} \mathrm{C}$ after 7 days. After 14 days, a superficial ring is also present. Teleomorph not observed.

Glucose is not fermented. Positive growth on D-glucose, D-galactose, D-ribose, D-xylose, Larabinose (weak), D-arabinose (delayed), sucrose (delayed), maltose, trehalose, methyl-alphaD-glucoside, cellobiose, salicin, melibiose, lactose, raffinose, melezitose, soluble starch, glycerol, erythritol, ribitol, xylitol, D-glucitol, D-mannitol, galactitol, inositol, methanol, ethanol, hexadecane, N-acetyl-D-glucosamine and ethyl acetate. No growth on L-sorbose, Lrhamnose, arbutin, glucono-delta-lactone, D-gluconate, D-glucoronate, D-galacturonate, DLlactate, succinate, citrate and L-malate. Utilisation of nitrogen sources: positive growth on potassium nitrate, sodium nitrite, ethylamine, lysine and cadaverine (weak). Growth in the presence of $0.01 \%$ is negative. Growth in the presence of $10 \% \mathrm{NaCl}$ and $50 \%$ glucose is negative. Growth on vitamin-free medium is positive. Urea hydrolysis and Diazonium Blue B reaction are positive. Starch-like compounds are produced. Maximum growth temperature: 25 ${ }^{\circ} \mathrm{C}$.

Molecular characteristics (holotype): nucleotide sequences of ITS and LSU (D1/D2 domains) rRNA deposited in NCBI/EMBL (GenBank) under the accession numbers: MK070337 and KC433768, respectively.

Deposits: holotype, strain MB 10.12 isolated from snow collected in July 2010 on Glacier du Geant, Mont Blanc massif, Alps, Italy (45.833333N, 6.916667E), preserved in a metabolically inactive state in the Industrial Yeasts Collection DBVPG, Department of Agricultural, Food and Environmental Sciences, University of Perugia, Perugia, Italy as DBVPG $5693^{\mathrm{T}}$. 
Strains studied: DBVPG 5693 ${ }^{\mathrm{T}}$, paratype DBVPG 5706 from the same locality.

994

995

Microbotryomycetes

996

Description of Yurkovia nerthusi Yurkov \& Begerow, sp. nov. (MB 828552)

997

Etymology: The specific epithet nerthusi is derived from a Latinized form (Nerthus) (NL.

998 gen. sing. m. n., snowy) of Germanic goddess of earth and fertility Nerpuz.

After growth on YM agar plates for $1 \mathrm{mo}$ at $16{ }^{\circ} \mathrm{C}$ and $22^{\circ} \mathrm{C}$, the streak culture is off-white to tan, butyrous to mucoid with a smooth and glistening surface. The margin is entire or rarely wrinkled. After growth on YM agar plates for $7 \mathrm{~d}$ at $16{ }^{\circ} \mathrm{C}$ and $22{ }^{\circ} \mathrm{C}$, cells are ellipsoidal or cylindrical (2-4 × 8-10 $\mu \mathrm{m})$, occurring singly or in pairs, and proliferating by polar budding. Pseudohyphae and true hyphae were not observed after 1 mo in Dalmau plate culture on CMA at $16-22^{\circ} \mathrm{C}$. Fermentation is absent.

Assimilation of carbon compounds: Growth on D-glucose, D-galactose, L-sorbose, Larabinose (weak), D-arabinose (weak), sucrose, maltose, trehalose, cellobiose, melezitose, inulin, starch, glycerol, ribitol, DL-lactate, succinate, citrate, ethanol (weak), L-tartaric acid, D-saccharic acid, 3,4-dyhydroxybesoic acid and 4-hydroxybencoic acid. No growth on raffinose, D-ribose, L-rhamnose, salicin, melibiose, lactose, erythritol, xylitol, galactitol and myo-inositol. Assimilation of nitrogen compounds: growth on potassium nitrate, sodium nitrite, ethylamine and lysine. Growth in the presence of $0.01 \%$ cycloheximide is positive. Growth in in the presence of $10 \% \mathrm{NaCl}$ and in medium with $50 \%$ and $60 \%$ glucose is negative. Urea hydrolysis and Diazonium Blue B reaction are positive. Maximum growth temperature: $25^{\circ} \mathrm{C}$.

Molecular characteristics (holotype): nucleotide sequences of LSU (D1/D2 domains) rRNA gene and ITS deposited in NCBI/EMBL (GenBank) under the accession number FN428970 and KY083054, respectively.

1018 Deposits: holotype, strain HEW-2-3 isolated from spruce forest soil collected in Stadtwald Mühlhausen, Thuringia, Germany $(51.21 \mathrm{~N}, 10.37 \mathrm{E})$, preserved in a metabolically inactive state in the German Collection of Microorganisms and Cell Cultures (DSMZ), Braunschweig, Germany as DSM 26788 ${ }^{\mathrm{T}}$. Ex-type cultures are deposited in the CBS yeast collection of the Westerdijk Fungal Biodiversity Institute, Utrecht, the Netherlands (CBS 11560) and the Mycothèque de l’Université Catholique de Louvain (BCCM/MUCL), Louvain-la-Neuve, 1024 Belgium (MUCL 53962).

1025 Strains studied: DSM DSM 26788 ${ }^{\mathrm{T}}$; paratype AEG-2-20 (= CBS 11560) isolated from grassland soil in Swabian Alb, in the proximity of Sternberg Wanderheim, Gomadingen, Baden-Wuerttemberg, Germany (48.391389N, 9.376889E); GenBank FN428969, KY083054.

1028 Notes: Strain AEG-2-20 was recovered on modified Browns' nitrogen deficient media 1029 (Yurkov et al. 2011), whereas strain HEW-2-3 was isolated on nutrient-rich acidified with 1030 lactic acid YPD agar (Yurkov et al. 2012a). 
1p32

1 b3

1034

1035

1 b36

1037

1038

1 b39

1040

1041

1042

1043

1044

1045

1046

1047

1048

1049

1050

1051

1052

1053

1054

1055

1056

1057

1058

1059

1060

1061

1062

1063

1064

1065

1066

1067

1068

1069

1 p70

Validation of Leucosporidium drummii Yurkov, A.M. Schäfer \& Begerow, International Journal of Systematic and Evolutionary Microbiology 62: 730 (2012); MB 563455

Leucosporidium drummii was described from soil by Yurkov et al. (2012b). However, the description does not conform to the ICN Melbourne Code Article 40.7, which requires that the single herbarium or collection or institution in which the type is conserved must be specified. We here fulfil the requirements for valid publication of Leucosporidium drummii.

Holotype: strain DSM $106046^{\mathrm{T}}$, isolated from grassland soil collected near Günterberg, Angermünde, Brandenburg, Germany, preserved in a metabolically inactive state in the German Collection of Microorganisms and Cell Cultures (DSMZ), Braunschweig, Germany. Ex-type cultures are deposited in the CBS yeast collection of the Westerdijk Fungal Biodiversity Institute, Utrecht, the Netherlands (CBS 11562) and the Mycothèque de l’Université Catholique de Louvain (BCCM/MUCL), Louvain-la-Neuve, Belgium (MUCL 52878).

Validation of Leucosporidium krtinense Mašínová, A. Pontes, C. Carvalho, J.P. Samp. \& Baldrian, International Journal of Systematic and Evolutionary Microbiology 67: 904 (2017); MB 815370

Leucosporidium krtinense was described from soil by Mašínová et al. (2017b). However, the description does not conform to the ICN Melbourne Code Article 40.7, which requires that the single herbarium or collection or institution in which the type is conserved must be specified. We here fulfil the requirements for valid publication of Leucosporidium krtinense. Holotype: strain PYCC 6879 isolated from beech litter collected in October 2013 in the Krrtiny forest area (49.303278N, 16.747389E), Czech Republic, preserved in a metabolically inactive state in the Portuguese Yeast Culture Collection, Caparica, Portugal. Ex-type cultures are deposited in the CBS yeast collection of the Westerdijk Fungal Biodiversity Institute, Utrecht, the Netherlands (CBS 14304) and the in the German Collection of Microorganisms and Cell Cultures (DSMZ), Braunschweig, Germany (DSM 101892).

Validation of Libkindia masarykiana Mašínová, A. Pontes, C. Carvalho, J.P. Samp. \& Baldrian, International Journal of Systematic and Evolutionary Microbiology 67: 906 (2017); MB 815373

Libkindia masarykiana was described from soil by Mašínová et al. (2017b). However, the description does not conform to the ICN Melbourne Code Article 40.7, which requires that the single herbarium or collection or institution in which the type is conserved must be specified. We here fulfil the requirements for valid publication of Libkindia masarykiana.

Holotype: strain PYCC 6886 ${ }^{\mathrm{T}}$ isolated from oak litter collected in April 2014 in the Kr̆tiny forest area (49.266944N, 16.721167E), Czech Republic, preserved in a metabolically inactive state in the Portuguese Yeast Culture Collection, Caparica, Portugal. Ex-type cultures are deposited in the CBS yeast collection of the Westerdijk Fungal Biodiversity Institute, Utrecht, 
1 the Netherlands (CBS 14275) and the in the German Collection of Microorganisms and Cell
1 th72 Cultures (DSMZ), Braunschweig, Germany (DSM 101891).

1073

1074

1075

1076

Validation of Yurkovia mendeliana Mašínová, A. Pontes, C. Carvalho, J.P. Samp. \& Baldrian, International Journal of Systematic and Evolutionary Microbiology 67: 907 (2017); MB 815372

Yurkovia mendeliana was described from soil by Mašínová et al. (2017b). However, the description does not conform to the ICN Melbourne Code Article 40.7, which requires that the single herbarium or collection or institution in which the type is conserved must be specified. We here fulfil the requirements for valid publication of Yurkovia mendeliana.

Holotype: strain PYCC $6884^{\mathrm{T}}$ isolated from beech litter collected in October 2013 in the Křtiny forest area (49.324944N, 16.652750E), Czech Republic, preserved in a metabolically inactive state in the Portuguese Yeast Culture Collection, Caparica, Portugal. Ex-type cultures are deposited in the CBS yeast collection of the Westerdijk Fungal Biodiversity Institute, Utrecht, the Netherlands (CBS 14273) and the in the German Collection of Microorganisms and Cell Cultures (DSMZ), Braunschweig, Germany (DSM 101889).

\section{Cystobasidiomycetes}

1089 CyphobasidialesT. Sprib. \& H. Mayrhofer (2016) Index Fungorum 309: 1 (MB 552589) nomen dubium

1091 The order Cyphobasidiales T. Sprib. \& H. Mayrhofer (2016) and family Cyphobasidiaceae T. Sprib. \& H. Mayrhofer (2016) were proposed to accommodate lichenicolous parasite Cyphobasidium Millanes, Diederich \& Wedin (2016) and lichen-inhabiting fungi represented by environmental sequences (Spribille et al. 2016). The monophyly of Cyphobasidium was not supported in the ML analysis of rRNA genes by Millanes et al. (2016). The analysis was also lacking Cyrenella elegans, which is the closest relative of Cyphobasidium according to sequence similarities from NCBI Blast and our phylogenetic analysis (Fig. 2). According to Bauer et al. (2006) Cyrenella elegans is placed in order Erythrobasidiales R. Bauer, Begerow, J.P. Samp., M. Weiss \& Oberw. (2006). The order Cyphobasidiales coincides with Erythrobasidiales and the latter is older name for the order. Although the principle of taxonomic priority does not apply above the rank of family (ICN Shenzhen Code, Art. 11.10), the name Cyphobasidiales T. Sprib. \& H. Mayrhofer (2016) is a duplicate and nomen dubium. Its delimitation from the order Erythrobasidiales is unclear and was not supported in our analyses. The order Erythrobasidiales includes genera Bannoa, Buckleyzyma, Cyrenella, Hasegawazyma, Erythrobasidium and Symmetrospora (Aime et al. 2006; Bauer et al. 2006; Wang et al. 2015a, 2015b). Erythrobasidium is an older generic name (ICN Shenzhen Code, Recommendation 16A.1) and the order Erythrobasidiales has been widely used by yeast taxonomists, mycologists and botanists (e.g. Aime et al. 2006; Bauer et al. 2006; Ruggiero et al. 2015; Oberwinkler 2017; Zhao et al. 2017). In our opinion, the name Cyphobasidiales should not be used in favour of the name Erythrobasidiales. 
Similarly, the proposal of the family CyphobasidiaceaeT. Sprib. \& H. Mayrhofer (2016) is premature since its delimitation from Buckleyzymaceae Q.M. Wang, F.Y. Bai, M. Groenew. \& Boekhout (2015), Erythrobasidiaceae Denchev (2009) and Symmetrosporaceae Q.M. Wang, F.Y. Bai, M. Groenew. \& Boekhout (2015) was not supported by a robust phylogenetic analysis.

\section{Discussion}

Two important evolutionary characters, such as the ability of basidiospores to germinate by secondary spore formation and active spore discharge ${ }_{2}$ connected yeasts with some Basidiomycetes (reviewed by Oberwinkler 1987). The evolution of filamentous habit was essential for penetration and growth in the substrate and production of fruiting bodies as well as for the emergence specialised organs like haustoria and sporangia. Although basidiomycetous yeast species with a sexual state do not form macroscopic fruiting bodies (basidiocarps) in culture, filaments and haustoria have been observed in several yeasts. Filamentous heterobasidiomycetes display diverse germination patterns, including budding, secondary spores, microconidia and hyphae. A close connection between filamentous parasites like Carcinomyces, Syzygospora and Tremella, and teleomorphic yeasts like Filobasidium and Filobasidiella (presently Cryptococcus), has been suggested based on the basidial morphology, presence of haustoria and on ultrastructural markers (Bandoni 1987; Oberwinkler 1987). The first molecular studies already confirmed close relationships between some filamentous Heterobasidiomycetes and yeasts, and subsequent works demonstrated that many basidiomycetous yeasts represent cultivable morphs of Heterobasidiomycetes presently classified in the classes Cystobasidiomycetes, Microbotryomycetes and Tremellomycetes (Boekhout et al. 1993; Fell et al. 2000; Scorzetti et al. 2002; Sampaio 2004; Bauer et al. 2006; Millanes et al. 2011; Liu et al. 2015a; Wang et al. 2015b). Also, large filamentous genera Tremella, Sirobasidium, Syzygospora and Cystobasidium were shown to be polyphyletic. The implementation of rRNA and multi-gene phylogenies has provided valuable information for the re-evaluation of the classification of these fungi (Liu et al. 2015a; Wang et al. 2015b). However, despite substantial progress in species sequencing, taxon sampling for teleomorphic taxa is meagre. Very few sequences were obtained for species of genera like Carcinomyces, Holtermannia, Sirobasidium, Sirotrema, Syzygospora, Tetragoniomyces and several lineages of Tremella. Sequences of type species of genera like Mycogloea, Occultifur, Sirobasidium and Spiculogloea are lacking. No sequences are available for Phragmoxenidium, Phyllogloea, Sigmogloea, Sigmotrema, Tremellina, Xenolachne and Zygogloea. Unfortunately, few culture experiments have been carried out to isolate and preserve these fungi. In addition, many species are rarely collected. New collections studied molecularly and preserved ex-situ would improve our understanding of the diversity and systematics of this group. The recent reassessment of the genus Phaeotremella provides a good example of the relevance of such taxonomic studies. Along with the re-collection the availability of cultures, isolates from several Tremella species collected by Franz Oberwinkler and his co-workers were authenticated and re-sequenced (Spirin et al. 2018). Another recent study by Pontes et al. (2017) studied the culture of Tetragoniomyces uliginosus, which was earlier obtained by 
1153 Franz Oberwinkler (PYCC database). Nucleotide sequences from this culture suggest that the

1154 phylogenetic placement of this species in the Trichosporonales (Liu et al. 2015a) is likely to

1155 be an artefact of a short sequence, which was originally obtained from a herbarium material

1156 (Millanes et al. 2011).

1157 Our phylogenetic analyses included sequences from single strains from different studies.

1158 Interestingly, some of them represent conspecific isolates, e.g. in genera Cryptococcus,

1159 Heterocephalacria, Piskurozyma and Pseudotremella (Figs. 1, 4, 5). A number of potential

1160 new species were shown to enlarge small or previously monotypic genera, e.g. Carcinomyces,

1161 Fibulobasidium, Gelidatrema, Heterocephalacria, Sugitazyma and Yurkovia (Figs. 1, 2). This

1162 observation legitimises to some extent the re-classification of large polymorphic genera into

1163 smaller, sometimes monotypic entities. A considerable number of species awaiting

1164 description would be otherwise assigned to genera Bensingtonia, Bullera, Cryptococcus,

1165 Rhodotorula, and Sporobolomyces, increasing taxonomic complexity of many groups. Newly

1166 erected genera of Microbotryomycetes accommodated species previously classified as

1167 Bensingtonia, Rhodotorula, and Sporobolomyces and ${ }_{2}$ in some cases, closely related species

1168 were assigned to different genera (Wang et al. 2015a, 2015b). Similarly, GenBank sequences

1169 of yeasts belonging to such problematic groups were mislabelled or had a doubtful taxonomic

1170 assignment up to the rank of a family. This situation is often confusing; it does not facilitate

1171 description of new species nor does it help to identify them in the environment. Sequences

1172 obtained from Genbank and a few other repositories were placed into the two LSU rRNA

1173 datasets used by Liu et al. (2015a) and Wang et al. (2015b) in order to provide an update on

1174 phylogenetic relationships in Tremellomycetes, Cystobasidiomycetes and

1175 Microbotryomycetes. The presented phylogenetic analyses (Figs. 1, 2) show the diversity of

1176 basidiomycetous yeasts and provide authors of potential new species with a robust

1177 phylogenetic analysis to ease future species descriptions. Discovery of a single strain

1178 representing a new species seldom warrants a publication. However, public sequences

1179 representing yet undescribed species become an extremely important source of rare fungi and

1180 urge researchers to release unpublished data of potential new species to facilitate their formal

1181 description. In the present study, sharing sequence data helped us to identify many closely

1182 related and conspecific strains originating from a number of independent studies.

1183

\section{Rare and rarely sampled}

1185 Yeasts thrive in the environment not as pure cultures but as a part of a microbial community

1186 which varies in time and space. Physico-chemical characteristics of the environment,

1187 substrate colonisation, dissemination of propagules, competition with other species, and

1188 interactions with potential vectors and predator-prey relationships determine composition of

1189 the community (reviewed in Yurkov 2017; Yurkov and Pozo 2017). As a result, most of

1190 known yeast communities consist of a few dominating species and a large number of species

1191 which are found in low numbers. While isolation of dominant species is easy to achieve,

1192 cultivation of less numerous species requires a larger sampling and cultivation effort.

1193 Consequently, it is well documented and understandable that some species are recovered from 
1194 the environment as single strains. These yeasts represent rare species, which are also rarely

1195 sampled.

1196 Yeasts used in this study were isolated from different substrates and regions, suggesting that basidiomycetous yeasts are rather widespread and not restricted to a specific location or substrate. Recent surveys of soils and cold habitats reported a substantial proportion of potential new yeasts (Buzzini et al. 2017; Sannino et al. 2017). These habitats are often characterized by very uneven structure of yeast communities with a few dominating species and a large number of rare yeasts. Most of the new soil-borne basidiomycetes recently described from soils represent rare taxa (e.g. Yurkov et al. 2016a, 2016b; Mašínová et al. 2017a, 2017b; Pontes et al. 2017). Mediterranean plants sampled in Serra da Arrábida (Portugal) during years 1997-1999 yielded diverse yeast communities, including many new species known from very few isolates (Inacio 2003). A number of these yeasts remained undescribed and were not found again until the recent resampling of the same biotopes

\section{7 (Yurkov et al. 2016b).}

1208 The number of known basidiomycetous species has increased dramatically in recent years. It is important to highlight that a number of species and genera in some groups were described from a very few strains and that this trend has increased in the last few years. For example,

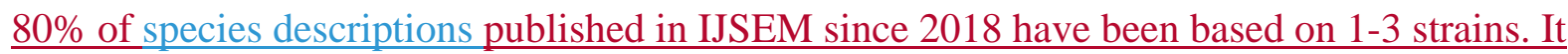
is also important to mention that taxonomic publications often do not provide essential information on the number of samples, isolated strains, species abundance or sampling depth. Therefore, it is not possible to conclude whether the sampling effort was sufficient in the aforementioned studies and these novel yeasts are rare species. Descriptions of either prokaryotic or eukaryotic species are expected to include a careful examination of species properties and ecology, which is difficult (if possible at all) to achieve on a limited number of strains. In our opinion, publication policies need to be modified to ensure that descriptions of novel taxa, especially genera, include (considering availability of both the material and data) all related strains and sequences.

Non-pigmented Microbotryomycetes, previously classified in the genera Bensingtonia, Rhodotorula and Sporobolomyces, are slow-growing species that are rarely isolated from the environment. Some species and genera are psychrophilic which makes their cultivation more complicated. During our past studies, we have also observed yeasts which grow slowly after isolation but lose their viability after a few passages. Therefore, fast and efficient preservation of yeast cultures is essential to keep the material for further investigations. Public databases, GenBank and culture collections contain nucleotide sequences of potential novel species which were isolated long time ago but await a formal description. Despite ongoing studies of European forest soils, no additional strain of species recently described by Yurkov et al. (2016a) such as Colacogloea, Hamamotoa and Slooffia were recovered even though they were isolated in 2008. Likewise, Yurkovia nerthusi described here is known from only two strains, and no additional culture of this species was found since the first isolation. However, several yeasts isolated by Fonseca et al. (2000) and Inacio (2003) from the Nature Park of Arrábida, were not found elsewhere. Heterocephalacria (formerly Cryptococcus) arrabidensis was isolated from plants and more species of this genus were identified and 
1236 described in this study (Figs. 1, 4). One of them is represented by two strains isolated from the 1237 same locality with a gap of 20 years. Similarly, Cystofilobasidium alribaticum was described

1238 after a recent resampling in Serra da Arrábida (Pontes et al. 2016). These examples show that 1239 rare species can persist in the environment for a long period. However, since these yeasts are

1240 rare and cultured as single isolates, a substantial sampling effort is required to resample them 1241 or to isolate them from a new source. We do not call for vast species descriptions based on a 1242 single culture but it is important to note that an effort to obtain additional isolates of some 1243 yeasts can be far too difficult.

\section{Acknowledgements}

1246 We owe much of our present knowledge of diversity and systematics of so-called dimorphic basidiomycetes heavily relies on specimens, sequence data and publications to Professor Franz Oberwinkler and his collaborators, including students and trainees of the Tübingen 1249 School.

1250 Álvaro Fonseca is much acknowledged for assistance in sampling in Arrábida Natural Park 1251 and analysis of yeasts from this locality. Angela Schäfer (RUB), Julian Federici (RUB) and 1252 Gabrielle Gresenz (DSMZ) are acknowledged for assistance in the lab. Two anonymous reviewers are acknowledged for their valuable comments on the manuscript. We thank MycoBank curator Dr. Konstanze Bensch for her advice on Latin names and Dr. Lara Barnes (School of Pharmacy and Biomolecular Sciences, University of Brighton) for correcting the English text.

Ana Pontes, Claudia Carvalho and José Paulo Sampaio acknowledge funding from FCT grant UID/Multi/04378/2013 (Portugal).

\section{References}

1261 Babjeva IP, Lisichkina GA, Reshetova IS, Danilevich VN (2002) Mrakia curviuscula sp.

1262 nov.: a new psychrophilic yeast from forest substrates. Microbiology (Moscow) 71:449-454

1263 Bandoni RJ (1979). Fibulobasidium: a new genus in the Sirobasidiaceae. Can J Bot 57:2641264268

1265 Bandoni RJ (1987) Taxonomic overview of the Tremellales. In: de Hoog GS, Smith MTh, 1266 Weijman ACM (eds), The Expanding Realm of Yeast-like Fungi. Elsevier, Amsterdam, pp $1267 \quad 87-110$

1268 Banno I (1963) Preliminary report on cell conjugation and mycelial stage in Rhodotorula 1269 yeasts. J Gen Appl Microbiol 9:249-251

1270 Banno I (1967) Studies on the sexuality of Rhodotorula. J Gen Appl Microbiol 13:167-196

1271 Bauer R, Begerow D, Sampaio JP, Weiß M, Oberwinkler F (2006) The simple-septate 1272 basidiomycetes: a synopsis. Mycol Prog 5:41-66 
1273 Begerow D, Bauer R, Oberwinkler F (1997) Phylogenetic studies on nuclear large subunit

1274 ribosomal DNA sequences of smut fungi and related taxa. Can J Bot 75:2045-2056

1275 Begerow D, Kemler M, Feige A, Yurkov A (2017) Parasitism in yeasts. In: Buzzini P, 1276 Lachance MA, Yurkov A (eds) Yeasts in Natural Ecosystems: Ecology. Springer, Cham, pp $1277 \quad 179-210$

1278 Boekhout T, Bandoni RJ, Fell JW, Kwon-Chung KJ, Sampaio JP, Fonseca Á (2011)

1279 Discussion of teleomorphic and anamorphic genera of heterobasidiomycetous yeasts. In:

1280 Kurtzman CP, Fell JW, Boekhout T (eds) The yeasts, a taxonomic study, 5th edn. Elsevier, 1281 Amsterdam, pp 1339-1374

1282 Boekhout T, Fonseca Á, Sampaio JP, Golubev WI (1993) Classification of

1283 heterobasidiomycetous yeasts: characteristics and affiliation of genera to higher taxa of

1284 Heterobasidiomycetes. Can J Microbiol 39:276-290

1285 Buzzini P., Turk M, Perini L, Turchetti B, Gunde-Cimerman N (2017) Yeasts in Polar and

1286 Subpolar Habitats. In: Buzzini P, Lachance MA, Yurkov A (eds) Yeasts in Natural

1287 Ecosystems: Diversity. Springer, Cham, pp 331-365

1288 Cadez N, Zupan J, Raspor P (2010) The effect of fungicides on yeast communities associated 1289 with grape berries. FEMS Yeast Res 10:619-630

1290 Clement M, Posada D, Crandall K (2000) TCS: a computer program to estimate gene 1291 genealogies. Mol Ecol 9:1657-1660

Dämon W, Hausknecht A (2002) First report of a Sirobasidium species in Austria, and a

1293 survey of the Sirobasidiaceae. Österr Z Pilzk 11:133-151 de García V, Brizzio S, Russo G, Rosa CA, Boekhout T, Theelen B, Libkind D, van Broock M (2010) Cryptococcus spencermartinsiae sp. nov., a basidiomycetous yeast isolated from glacial waters and apple fruits. Int J Syst Evol Microbiol 60:707-711

Endoh R, Suzuki M, Okada G, Takeuchi Y, Futai K (2011) Fungus symbionts colonizing the galleries of the ambrosia beetle Platypus quercivorus. Microb Ecol 62:106-120

Fell JW, Boekhout T, Fonseca Á, Scorzetti G, Statzell-Tallman A (2000) Biodiversity and systematics of basidiomycetous yeasts as determined by large-subunit rDNA D1/D2 domain sequence analysis. Int J Syst Evol Microbiol 50:1351-1372

1302 Fell JW, Boekhout T, Freshwater DW (1995) The role of nucleotide sequence analysis in the 1303 systematics of the yeast genera Cryptococcus and Rhodotorula. Stud Mycol 38:129-146

1304 Fonseca Á, Boekhout T, Fell JW (2011). Cryptococcus Vuillemin (1901). In: The yeasts: a 1305 taxonomic study (Kurtzman CP, Fell JW, Boekhout T, eds). Elsevier, Amsterdam, pp 1661$1306 \quad 1737$

1307 Fonseca Á, Scorzetti G, Fell JW (2000) Diversity in the yeast Cryptococcus albidus and 1308 related species as revealed by ribosomal DNA sequence analysis. Can J Microbiol 46:7-27 
1309 Fotedar R, Kolecka A, Boekhout T, Fell JW, Anand A, Al Malaki A, Zeyara A, Al Marri M

1310 (2018) Naganishia qatarensis sp. nov., a novel basidiomycetous yeast species from a

1311 hypersaline marine environment in Qatar. Int J Syst Evol Microbiol 68:2924-2929ю

1312 França L, Sannino C, Turchetti B, Buzzini P, Margesin R (2016) Seasonal and altitudinal

1313 changes of culturable bacterial and yeast diversity in Alpine forest soils. Extremophiles

$1314 \quad 20: 855-873$

1315 Glushakova AM, Kachalkin AV (2017) Endophytic yeasts in Malus domestica and Pyrus

1316 communis fruits under anthropogenic impact. Microbiology (Moscow) 86:128-135

1317 Glushakova AM, Kachalkin AV, Chernov IY (2015) Effect of invasive herb species on the

1318 structure of soil yeast complexes in mixed forests exemplified by Impatiens parviflora DC.

1319 Microbiology (Moscow) 84:717-721

1320 Herzberg M, Fischer R, Titze A (2002) Conflicting results obtained by RAPD-PCR and large-

1321 subunit rDNA sequences in determining and comparing yeast strains isolated from flowers: a

1322 comparison of two methods. Int J Syst Evol Microbiol. 52:1423-1433

1323 Inácio J (2003) Yeast occurrence and diversity on the phylloplane of selected plants from the

1324 Arrábida Natural Park. PhD thesis (in Portuguese). Universidade Nova de Lisboa, Portugal

1325 Inácio J, Pereira P, de Carvalho M, Fonseca Á, Amaral-Collaço MT, Spencer-Martins I

1326 (2002) Estimation and diversity of phylloplane mycobiota on selected plants in a

1327 Mediterraneantype ecosystem in Portugal. Microb Ecol 44:344-353

1328 Kachalkin AV, Glushakova AM, Yurkov AM, Chernov IYu (2008) Characterization of yeast

1329 groupings in the phyllosphere of sphagnum mosses. Microbiology (Moscow) 77:474-481

1330 Katoh K, Rozewicki J, Yamada KD (2017) MAFFT online service: multiple sequence

1331 alignment, interactive sequence choice and visualization. Brief Bioinform bbx108

1332 Kluyver AJ, van Niel CB (1927). Sporobolomyces: ein Basidiomyzet? Ann Mycol 25:389-

$1333 \quad 394$

1334 Kurtzman CP, Boekhout T (2017) Yeasts as distinct life forms of Fungi. In: Buzzini P,

1335 Lachance MA, Yurkov A (eds) Yeasts in Natural Ecosystems: Ecology. Springer, Cham, pp

$1336 \quad 1-37$

1337 Kurtzman CP, Fell JW, Boekhout T, Robert V (2011) Methods for isolation, phenotypic

1338 characterization and maintenance of yeasts. In: Kurtzman CP, Fell JW, Boekhout T (eds) The

1339 yeasts, a taxonomic study, 5th edn. Elsevier, Amsterdam, pp 97-107

1340 Liu XZ, Wang QM, Göker M, Groenewald M, Kachalkin AV, Lumbsch HT, Millanes AM,

1341 Wedin M, Yurkov AM, Boekhout T, Bai FY (2015a) Towards an integrated phylogenetic

1342 classification of the Tremellomycetes. Stud Mycol 81:85-147

1343 Liu XZ, Wang QM, Theelen B, Groenewald M, Bai FY, Boekhout T (2015b) Phylogeny of

1344 tremellomycetous yeasts and related dimorphic and filamentous basidiomycetes reconstructed

1345 from multiple gene sequence analyses. Stud Mycol 81:1-26. 
1346 Mašínová T, Bahnmann BD, Větrovský T, Tomšovský M, Merunková K, Baldrian P (2017a)

1347 Drivers of yeast community composition in the litter and soil of a temperate forest. FEMS

1348 Microbiol Ecol 93:fiw223

1349

1350

1351

1352

1353

1354

1355

1356

1357

1358

1359

1360

1361

1362

1363

1364

1365

1366

1367

1368

1369

1370

$1 \beta 71$

1372

1373

1374

1375

1376

1377

1378

1379

1380

1381

Mašínová T, Pontes A, Carvalho C, Sampaio JP, Baldrian P (2017b) Libkindia masarykiana gen. et sp. nov., Yurkovia mendeliana gen. et sp. nov. and Leucosporidium krtinense fa sp. nov., isolated from temperate forest soils. Int J Syst Evol Microbiol 67:902-908.

Mašínová T, Yurkov A, Baldrian P (2018) Forest soil yeasts: decomposition potential and the utilization of carbon sources. Fungal Ecol 34:10-19

Millanes AM, Diederich P, Ekman S, Wedin M (2011) Phylogeny and character evolution in the jelly fungi (Tremellomycetes, Basidiomycota, Fungi). Mol Phylogenet Evol 61:12-28.

Millanes AM, Diederich P, Wedin M (2016) Cyphobasidium gen. nov., a new licheninhabiting lineage in the Cystobasidiomycetes (Pucciniomycotina, Basidiomycota, Fungi). Fungal Biol 120:1468-1477

Mittelbach M, Yurkov AM, Stoll R, Begerow D (2016) Inoculation order of nectar-borne yeasts opens a door for transient species and changes nectar rewarded to pollinators. Fungal Ecol 22:90-97

Oberwinkler F (1987) Heterobasidiomycetes with ontogenetic yeast-stages - systematic and phylogenetic aspects. In: de Hoog GS, Smith MTh, Weijman ACM (eds), The Expanding Realm of Yeast-like Fungi. Elsevier, Amsterdam, pp 61-74

Oberwinkler F (2017) Yeasts in Pucciniomycotina. Mycol Progress 16:831-856

Péter G, Takashima M, Čadež N. (2017) Yeast habitats: different but global. In: Buzzini P, Lachance MA, Yurkov A (eds) Yeasts in Natural Ecosystems: Ecology. Springer, Cham, pp 39-71

Pontes A, Röhl O, Maldonado C, Yurkov AM, Sampaio JP (2017) Cryptotrichosporon argae sp. nov., Cryptotrichosporon brontae sp. nov. and Cryptotrichosporon steropae sp. nov., isolated from forest soils. Int J Syst Evol Microbiol 67:3610-3614

Ruggiero MA, Gordon DP, Orrell TM, et al. (2015) A higher level classification of all living organisms. PloS One 10:e0119248

Sampaio JP (2004) Diversity, phylogeny and classification of basidiomycetous yeasts. In:

Agerer R, Piepenbring M, Blanz P (eds), Frontiers in Basidiomycote Mycology. IHW Verlag, Eching, pp 49-80

Sampaio JP (2011) Rhodotorula Harrison (1928). In: Kurtzman CP, Fell JW, Boekhout T (eds) The yeasts: a taxonomic study, 5th edn. Elsevier, Amsterdam, pp 1873-1927

Sannino C, Tasselli G, Filippucci S, Turchetti B, Buzzini P (2017) Yeasts in Nonpolar Cold Habitats. In: Buzzini P, Lachance MA, Yurkov A (eds) Yeasts in Natural Ecosystems:

Diversity. Springer, Cham, pp 367-396 
1382 Scorzetti G, Fell JW, Fonseca Á, Statzell-Tallman A (2002) Systematics of basidiomycetous 1383 yeasts: a comparison of large subunit D1D2 and internal transcribed spacer rDNA regions.

1384 FEMS Yeast Res 2: 495-517

1385 Seifert KA, Rossman AY (2010) How to describe a new fungal species. IMA Fungus. 1:1091386116

1387 Selbmann L, Zucconi L, Onofri S, Cecchini C, Isola D, Turchetti B, Buzzini P (2014)

1388 Taxonomic and phenotypic characterization of yeasts isolated from worldwide cold rock1389 associated habitats. Fungal Biol 118:61-71

1390 Šibanc N, Zalar P, Schroers HJ, Zajc J, Pontes A, Sampaio JP, Maček I (2018) Occultifur 1391 mephitis f.a., sp. nov. and other yeast species from hypoxic and elevated CO2 mofette 1392 environments. Int J Syst Evol Microbiol. 68:2285-2298

1393 Spribille T, Tuovinen V, Resl P et al (2016) Basidiomycete yeasts in the cortex of ascomycete 1394 macrolichens. Science 353:488-492

1395 Spirin V, Malysheva V, Yurkov A et al (2018) Studies in the Phaeotremella foliacea group 1396 (Tremellomycetes, Basidiomycota). Mycol Prog 17:451-466

1397 Sylvester K, Wang QM, James B, Mendez R, Hulfachor AB, Hittinger CT (2015)

1398 Temperature and host preferences drive the diversification of Saccharomyces and other 1399 yeasts: a survey and the discovery of eight new yeast species. FEMS Yeast Res 15:fov002

1400 Taylor DL, McCormick MK (2008) Internal transcribed spacer primers and sequences for 1401 improved characterization of basidiomycetous orchid mycorrhizas. New Phytol 177:102014021033

1403 Turchetti B, Goretti M, Branda E, Diolaiuti G, D’Agata C, Smiraglia C, Onofri A, Buzzini P 1404 (2013) Influence of abiotic variables on culturable yeast diversity in two distinct Alpine 1405 glaciers. FEMS Microbiol Ecol 86:327-340

1406 Turchetti B, Selbmann L, Gunde-Cimerman N, Buzzini P, Sampaio JP, Zalar P (2018)

1407 Cystobasidium alpinum sp. nov. and Rhodosporidiobolus oreadorum sp. nov. from European 1408 Cold Environments and Arctic Region. Life 8:9

1409 Wang QM, Groenewald M, Takashima M, Theelen B, Han PJ, Liu XZ, Boekhout T, Bai FY 1410 (2015a) Phylogeny of yeasts and related filamentous fungi within Pucciniomycotina 1411 determined from multigene sequence analyses. Stud. Mycol 81:27-53

1412 Wang QM, Yurkov AM, Göker M, Lumbsch HT, Leavitt SD, Groenewald M, Theelen B, Liu 1413 XZ, Boekhout T, Bai FY (2015b) Phylogenetic classification of yeasts and related taxa within 1414 Pucciniomycotina. Stud Mycol 81:149-189

1415 Yurkov AM (2017) Temporal and Geographic Patterns in Yeast Distribution. In: Buzzini P, 1416 Lachance MA, Yurkov A (eds) Yeasts in Natural Ecosystems: Ecology. Springer, Cham, pp $1417 \quad 101-130$ 
Yurkov AM, Pozo MI (2017) Yeast Community Composition and Structure. In: Buzzini P, Lachance MA, Yurkov A (eds) Yeasts in Natural Ecosystems: Ecology. Springer, Cham, pp 73-100

1421 Yurkov AM, Kemler M, Begerow D (2012a) Assessment of yeast diversity in soils under different management regimes. Fungal Ecol 5:24-35

1423 Yurkov AM, Schäfer AM, Begerow D (2012b) Leucosporidium drummii sp. nov., a member of the Microbotryomycetes isolated from soil. Int J Syst Evol Microbiol 62:728-734.

Yurkov A, Guerreiro MA, Sharma L, Carvalho C, Fonseca Á (2015a) Multigene Assessment of the Species Boundaries and Sexual Status of the Basidiomycetous Yeasts Cryptococcus flavescens and C. terrestris (Tremellales). PLoS One 10:e0120400

1428 Yurkov AM, Kachalkin AV, Daniel HM, Groenewald M, Libkind D, de Garcia V, Zalar P, 1429 Gouliamova DE, Boekhout T, Begerow D (2015b) Two yeast species Cystobasidium psychroaquaticum f.a. sp. nov. and Cystobasidium rietchieii f.a. sp. nov. isolated from natural environments, and the transfer of Rhodotorula minuta clade members to the genus Cystobasidium. Antonie van Leeuwenhoek 107:173-185

1433 Yurkov AM, Wehde T, Federici J et al (2016a) Yeast diversity and species recovery rates from beech forest soils. Mycol Prog 15:845-859

Yurkov AM, Röhl O, Pontes A et al. (2016b) Local climatic conditions constrain soil yeast diversity patterns in Mediterranean forests, woodlands and scrub biome FEMS Yeast Res 16: fov103

Yurkov AM, Dlauchy D, Péter G (2017) Meyerozyma amylolytica sp. nov. from temperate deciduous trees and the transfer of five Candida species to the genus Meyerozyma. Int J Syst Evol Microbiol 67:3977-3981

Zhao RL, Li GJ, Sánchez-Ramírez S, et al. (2017). A six-gene phylogenetic overview of Basidiomycota and allied phyla with estimated divergence times of higher taxa and a phyloproteomics perspective. Fungal Divers 84:43-74.

Zhao Y, Liu XZ, Bai FY (2019) Four new species of Tremella (Tremellales, Basidiomycota) based on morphology and DNA sequence data. MycoKeys 47:75-95

\section{Figure captions}

1448 Figure 1 - Phylogenetic relationships of yeasts and related taxa in Agaricomycotina obtained 1449 by maximum-likelihood analysis of LSU (D1/D2 domains) rRNA gene. Tree topology was 1450 constrained according to the topology of the seven genes-based tree (for details see Liu et al. 1451 2015a, 2015b) with nodes showing bootstrap values >85 \% inforced to be monophyletic. Taxa 1452 not included in the previous analysis by Liu et al. (2015) are in red. The numbers provided on 1453 branches are frequencies (> $50 \%$ ) with which a given branch appeared in 100 bootstrap 1454 replications. The scale bars indicate the numbers of expected substitutions accumulated per 1455 site. 
1456 Figure 2 - Phylogenetic relationships of yeasts and related taxa from Pucciniomycotina 1457 lineages obtained by Maximum-Likelihood analysis of the LSU (D1/D2 domains) rRNA

1458 gene. Tree topology was constrained according to the topology of the seven genes-based tree 1459 (for details see Wang et al. 2015a, 2015b) with nodes showing bootstrap values $>85 \%$

1460 inforced to be monophyletic. Taxa not included in the phylogenetic analysis of the seven 1461 genes (Wang et al. 2015a) are indicated in red. The numbers provided on branches are 1462 frequencies (> $50 \%$ ) with which a given branch appeared in 100 bootstrap replications. The 1463 scale bars indicate the numbers of expected substitutions accumulated per site.

1464 Figure 3 - Phylogenetic relationships of yeasts and related taxa from the order

1465 Cystofilobasidiales in Tremellomycetes obtained by maximum-likelihood analysis of a 1466 concatenated alignment of the (A) ITS region and LSU (D1/D2 domains) rRNA gene and (B) 1467 ITS region, LSU rRNA gene and TEF1. The numbers provided on branches are frequencies 1468 (> 50 \%) with which a given branch appeared in 100 bootstrap replications. The scale bars 1469 indicate the numbers of expected substitutions accumulated per site.

1470 Figure 4 - Maximum likelihood analysis of a concatenated alignment of the ITS region and 1471 LSU (D1/D2 domains) rRNA gene for the genus Heterocephalacria. The numbers provided 1472 on branches are frequencies (> 50 \%) with which a given branch appeared in 100 bootstrap 1473 replications. The scale bars indicate the numbers of expected substitutions accumulated per 1474 site.

1475 Figure 5 - Maximum likelihood analysis of a concatenated alignment of the ITS region and 1476 LSU (D1/D2 domains) rRNA gene for the genus Piskurozyma. The numbers provided on 1477 branches are frequencies (> $50 \%$ ) with which a given branch appeared in 100 bootstrap 1478 replications. The scale bars indicate the numbers of expected substitutions accumulated per 1479 site.

1480 Figure 6 - Maximum likelihood analysis of a concatenated alignment of the ITS region and 1481 LSU (D1/D2 domains) rRNA gene for the genus Naganishia. The numbers provided on 1482 branches are frequencies (> $50 \%$ ) with which a given branch appeared in 100 bootstrap 1483 replications. The scale bars indicate the numbers of expected substitutions accumulated per 1484 site.

1485 Figure 7 -Micrographs showing morphology of new species: Vustinia terrae (a), vegetative 1486 1487 cells on PDA after $7 \mathrm{~d}$ at $20^{\circ} \mathrm{C}$; Udeniomyces caspiensis (b), vegetative cells on $2 \%$ Glucose

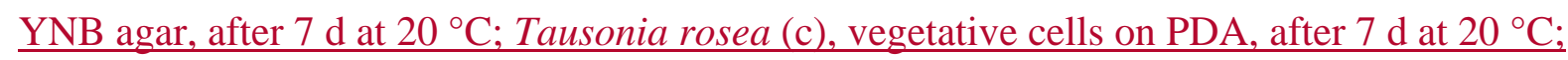
Udeniomyces orazovii (d-f), vegetative cells (d), microcolony (e), and chlamydospores (f) on PDA after $10 \mathrm{~d}$ at $20^{\circ} \mathrm{C}$; Itersonilia diksonensis (g, h), vegetative cells (g) and sympodially branched sterigmata with ballistoconidia (h) on $2 \%$ Glucose YNB agar, after $14 \mathrm{~d}$ at $20{ }^{\circ} \mathrm{C}$; Krasilnikovozyma fibulata (i-k), vegetative cells (i), hyphae with clamp connections and teliospores (j, k) on PDA after $14 \mathrm{~d}$ at $20^{\circ} \mathrm{C}$. Scale bars: $10 \mu \mathrm{m}$ (a, b, c, d, g, h, i, k), $20 \mu \mathrm{m}$ (f), $60 \mu \mathrm{m}$ (e) and $70 \mu \mathrm{m}$ (i). 